ARTICLE

\title{
IRF1-mediated downregulation of PGC1 $\alpha$ contributes to cardiorenal syndrome type 4
}

Yinghui Huang ${ }^{1,2}$, Shaobo Wang ${ }^{1}$, Jie Zhou ${ }^{3}$, Yong Liu', Changhong Du², Ke Yang ${ }^{1}$, Xianjin Bi ${ }^{1}$, Mingying Liu',

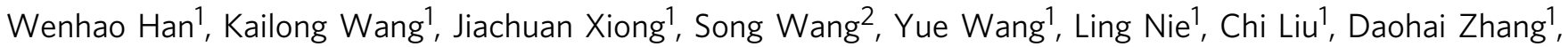
Jun $\mathrm{Gu}^{4}$, Chunyu Zeng (iD ${ }^{5} \&$ Jinghong Zhao (iD ${ }^{1 凶}$

Cardiorenal syndrome type 4 (CRS4) is a common complication of chronic kidney disease (CKD), but the pathogenic mechanisms remain elusive. Here we report that morphological and functional changes in myocardial mitochondria are observed in CKD mice, especially decreases in oxidative phosphorylation and fatty acid metabolism. High phosphate (HP), a hallmark of CKD, contributes to myocardial energy metabolism dysfunction by downregulating peroxisome proliferator-activated receptor gamma coactivator 1 alpha (PGC1 $\alpha$ ). Furthermore, the transcriptional factor interferon regulatory factor 1 (IRF1) is revealed as the key molecule upregulated by HP through histone H3K9 acetylation, and responsible for the HP-mediated transcriptional inhibition of PGC1 $\alpha$ by directly binding to its promoter region. Conversely, restoration of PGC1 $\alpha$ expression or genetic knockdown of IRF1 significantly attenuates HP-induced alterations in vitro and in vivo. These findings demonstrate that IRF1PGC1 $\alpha$ axis-mediated myocardial energy metabolism remodeling plays a crucial role in the pathogenesis of CRS4.

\footnotetext{
${ }^{1}$ Department of Nephrology, The Key Laboratory for the Prevention and Treatment of Chronic Kidney Disease of Chongqing, Kidney Center of PLA, Xinqiao Hospital, Army Medical University (Third Military Medical University), 400037 Chongqing, China. ${ }^{2}$ Institute of Combined Injury, State Key Laboratory of Trauma, Burns and Combined Injury, Chongqing Engineering Research Center for Nanomedicine, College of Preventive Medicine, Army Medical University, 400038 Chongqing, China. ${ }^{3}$ Department of Oncology and Southwest Cancer Center, Southwest Hospital, Army Medical University, 400038

Chongqing, China. ${ }^{4}$ State Key Laboratory of Protein and Plant Gene Research, College of Life Science, Peking University, 100871 Beijing, China. ${ }^{5}$ Department of Cardiology, Daping Hospital, Army Medical University, 400042 Chongqing, China. ${ }^{凶}$ email: zhaojh@tmmu.edu.cn
} 
C ardiovascular disease (CVD) is the most common complication of chronic kidney disease (CKD) and the leading cause of death (more than 50\%) in patients with $\mathrm{CKD}^{1,2}$. CKD may lead to pathologic cardiac changes including left ventricular hypertrophy (LVH), diastolic dysfunction, and/or increased risk of cardiovascular events, and this condition is called cardiorenal syndrome type 4 (CRS4) ${ }^{3}$. Astonishingly, up to $30 \%$ of CKD patients suffer from chronic heart failure $(\mathrm{HF})^{1,2}$, and about $40-50 \%$ of patients with HF have coexisting chronic renal dysfunction ${ }^{4}$. As reported, the risk of HF is much higher in patients with an estimated glomerular filtration rate (eGFR) $<60 \mathrm{ml} / \mathrm{min}$ per $1.73 \mathrm{~m}^{2}$, especially in dialysis patients ${ }^{2,5}$. However, the mechanism of increased susceptibility to HF in CKD patients remains elusive ${ }^{2,4}$.

As abundant ATP is required for normal systole and diastole, a severe decrease of ATP will directly disrupt cardiac function and eventually lead to $\mathrm{HF}^{6-8}$. Normally, over $95 \%$ of cardiac ATP is derived from oxidative phosphorylation (OXPHO), and the remaining $5 \%$ principally comes from glycolysis. The substrate utilization in the adult heart is mainly from the oxidation of fatty acid (FAO, $70-90 \%)$, glucose and lactate $(10-30 \%)^{7}$. Of note, FAO and OXPHO frequently decrease in a hypertrophic or failing heart, while glycolysis often compensatorily increases, known as myocardial energy metabolism remodeling ${ }^{7}$. This remodeling is considered responsible for the progression of $\mathrm{LVH}$ and $\mathrm{HF}^{8}$. A clinical study showed that a significant amelioration of cardiac function and reduction of the left ventricular mass index (LVMI) were observed in hemodialysis patients after treatment with Levocarnitine, a cofactor of FAO key enzyme carnitine palmitoyl transferase $1(\mathrm{Cpt} 1)^{9}$, indicating that severe disorder of myocardial energy metabolism may exist in patients with CKD. However, there is a lack of an in-depth study on the pathogenesis of CKD-associated myocardial energy metabolism dysfunction.

Traditional risk factors of CVD are insufficient to explain the high prevalence of HF in CKD patients ${ }^{10,11}$, such as hypertension, hemodynamic alterations, and activation of the renin-angiotensinaldosterone system (RAAS) and sympathetic nervous system. Therefore, increasing attention was paid to CKD-inherent risk factors, i.e. uremic toxins that accumulated in the body during disease progression. Retained uremic toxins can be divided into three groups according to physicochemical characteristics: small water-soluble compounds (molecular weight $(\mathrm{MW})<500 \mathrm{Da}$ ), middle-molecules (500 Da $<\mathrm{MW}<32,000 \mathrm{Da}$ ) and protein-bound small molecules (most of them with $\mathrm{MW}<500 \mathrm{Da}$ ). Remarkably, recent studies have reported that uremic toxins are not only a consequence of $\mathrm{CKD}$, but also a cause of uremic symptoms, including $\mathrm{CVD}^{1,2}$. Several water-soluble toxins have emerged as hallmarks of $\mathrm{CKD}^{12,13}$ and risk factors for CVD in CKD patients ${ }^{14}$, such as high phosphate (HP), fibroblast growth factor 23 (FGF23), and trimethylamine- $N$-oxide (TMAO). Middlemolecules can be used to predict the severity of coronary artery disease, such as $\beta 2$-microglobulin (B2M) ${ }^{15}$. Protein-bound toxins are associated with CKD progression and increased cardiovascular mortality, such as Indoxyl sulfate (IS) and $p$-cresyl sulfate (PCS $)^{16}$. Despite these findings, the distinct roles of uremic toxins in the pathogenesis of CRS4 and the underlying mechanisms are not fully understood.

In this study, we demonstrate that peroxisome proliferatoractivated receptor-gamma coactivator 1 alpha (PGC1a) is downregulated in the heart of CKD mice and PGC1a downregulation mediates myocardial energy metabolism remodeling that contributes to CKD-associated HF. HP is a crucial uremic toxin involved in myocardial energy metabolism dysfunction and could induce PGC1a downregulation. Moreover, the transcriptional factor interferon regulatory factor 1 (IRF1) mediates HP-induced PGC1a suppression by directly binding to its promoter region, thus generating HP-induced mitochondrial energy metabolism dysfunction and CKD-associated HF.

\section{Results}

Energy metabolism changes and PGC1a repression in CKD mice. To broadly explore the pathogenesis of CKD-associated CVD, we first examined genome-wide transcriptional changes in the heart of CKD mice by performing microarray analysis using Affymetrix Clariom S array. Gene ontology (GO) analysis revealed that the markedly downregulated genes were focused on mitochondrion (Fig. 1a). Kyoto Encyclopedia of Genes and Genomes (KEGG)-based pathway classification of the differentially expressed genes indicated significant changes in fatty acid metabolism (Fig. 1b). Meanwhile, transmission electron microscope (TEM) observation found mitochondrial derangements, swelling, and vacuolation with disrupted cristae in cardiomyocytes of CKD mice (Fig. 1c). Notably, microarray analysis showed that PGC1a, the key regulator of mitochondrial biogenesis and energy metabolism ${ }^{17,18}$, and its target genes were significantly inhibited in CKD mice (Fig. 1d). Then, quantitative real-time polymerase chain reaction (qPCR) and western blot verified that PGC1 $\alpha$ and its target genes [transcription factor A, mitochondrial (TFAM), estrogen-related receptor alpha (ERRa) and nuclear respiratory factor 1 (NRF1)], the genes related to OXPHO [ATP synthase, $\mathrm{H}+$ transporting, mitochondrial F1 complex, alpha subunit 1 (Atp5a1), NADH-ubiquinone oxidoreductase alpha subunit (Ndufa), NADH-ubiquinone oxidoreductase flavoprotein (Ndufv) and cytochrome $c$ (Cytc)] and the genes related to FAO [medium chain acyl-CoA dehydrogenase (MCAD), carnitine $O$ octanoyltransferase (CROT), hydroxyacyl-coenzyme A dehydrogenase $(\mathrm{HADHB})$ and $\mathrm{Cpt} 1 \mathrm{~b}]$ were significantly repressed in myocardial tissues from CKD mice (Fig. 1e-h). These data indicate that $\mathrm{CKD}$ is associated with myocardial energy metabolism dysfunction accompanied by PGC1a downregulation in cardiomyocytes.

Effects of HP on PGC1 a expression and energy metabolism. As the development of CKD-associated CVD is closely related to CKD-inherent risk factors ${ }^{19}$, we treated cardiac cells with uremic toxins to search for the causes of CKD-induced PGC1a downregulation and mitochondrial energy metabolism dysfunction. Interestingly, we observed that PGC1 $\alpha$ expression was not significantly suppressed by urea, serum creatinine ( $\mathrm{Scr}$ ), uric acid (UA), parathyroid hormone (PTH), TMAO, FGF23, B2M, IS, PCS, or indole-3-acetic acid (IAA), whereas HP had a strong ability to inhibit PGC1a expression (Fig. 2a). Moreover, PGC1a expression was also suppressed in cardiac cells incubated with the serum of CKD patients with hyperphosphatemia $(6.90 \pm 1.09 \mathrm{mg} /$ dl) (Fig. 2b, c). We then demonstrated that PGC1a expression was dose- and time-dependently downregulated by HP at both transcriptional and translational levels (Fig. $2 \mathrm{~d}-\mathrm{g}$ ). To investigate the role of HP in mitochondrial energy metabolism, we treated cardiac cells with HP and found suppression of oxygen consumption rate (OCR), decrease in ATP level, downregulation of FAO and OXPHO-related genes, upregulation of glycolysisrelated genes, reduction of mitochondrial DNA (mtDNA) copy number, the elevation of reactive oxygen species (ROS) production, and loss of mitochondrial membrane potential (Fig. $2 \mathrm{~h}-\mathrm{p}$ ). In addition, similar results including downregulated PGC1a expression and energy metabolism remodeling were observed in HP-incubated primary neonatal rat ventricular myocytes (NRVMs) (Supplementary Fig. 1A-G).

To explore the relationship between $\mathrm{HP}$ and $\mathrm{CKD}$-associated cardiac remodeling, 213 predialysis patients with eGFR of $2.00-23.30 \mathrm{ml} / \mathrm{min}$ per $1.73 \mathrm{~m}^{2}$ were enrolled in this study. The 
a

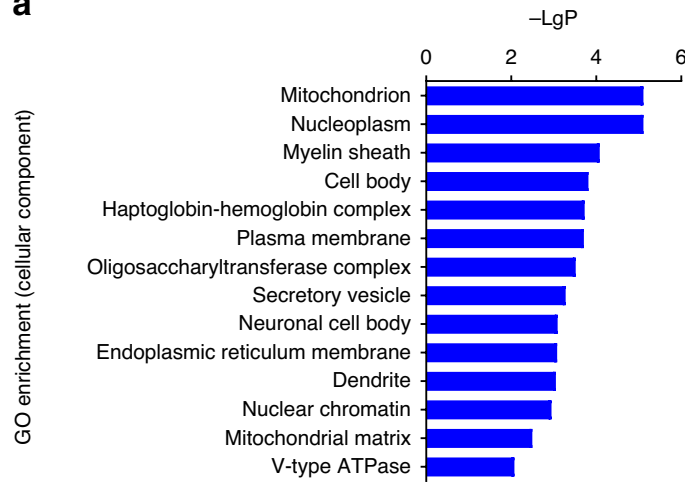

b

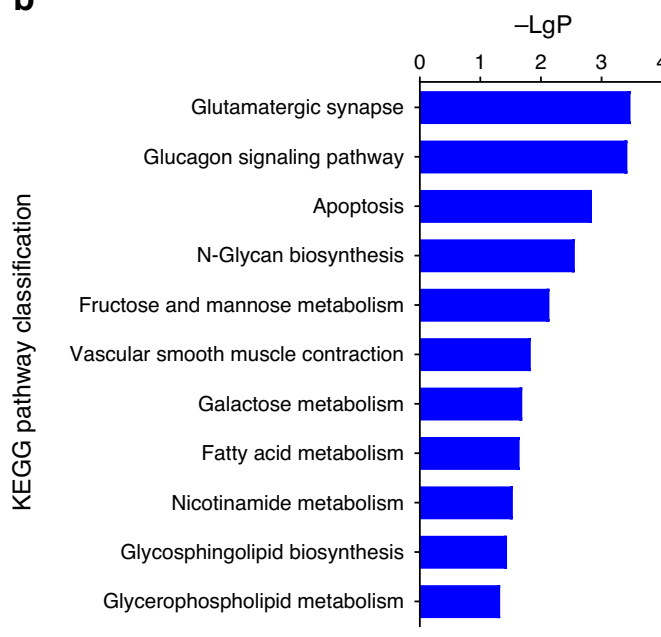

C

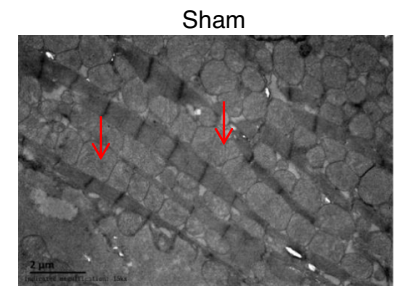

d

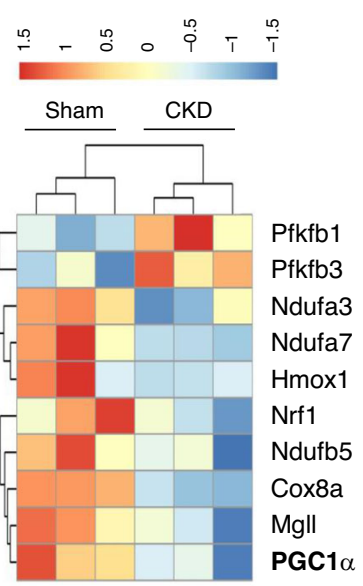

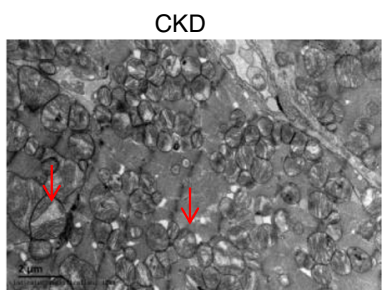
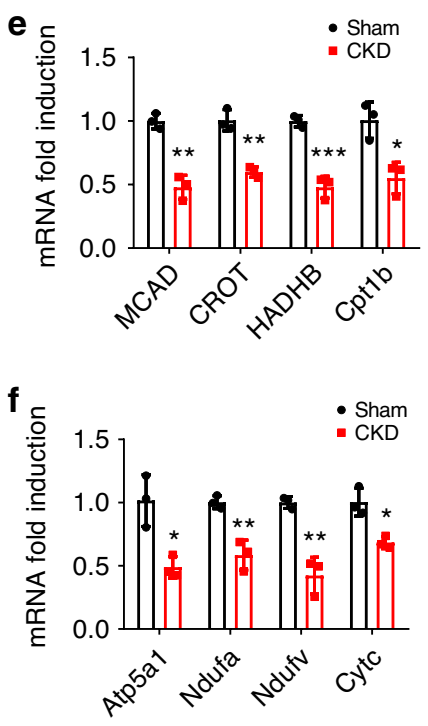

g

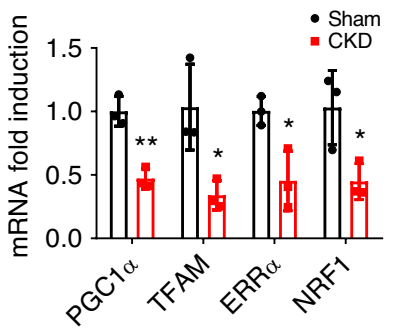

h

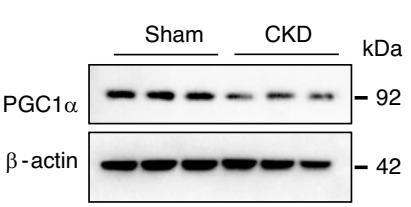

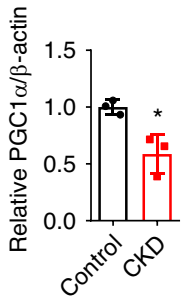

Fig. 1 Myocardial energy metabolism dysfunction and PGC1 $\alpha$ downregulation are observed in the heart of CKD mice. a GO enrichment of the significantly changed cellular components of the heart from three pairs of sham and CKD mice. $\mathbf{b}$ KEGG pathway classification of differentially expressed genes (DEG) of the heart in sham and CKD mice. c Representative images of transmission electron microscope (TEM) observation of mitochondria of the heart from sham and CKD mice ( $n=3$ mice per group). Scale bar, $2 \mu \mathrm{m}$. Red arrows point to mitochondria. $\mathbf{d}$ Heatmap of DEG (mainly PGC1 $\alpha$ and its target genes) of mice in $\mathbf{a}$. The color intensity represents row Z-score. Red color indicates highly expressed, while blue indicates lowly expressed. e-g Relative mRNA expression of FAO genes (e), OXPHO genes (f), and PGC1 $\alpha$ and its target genes (g) of the heart from sham and CKD mice. $\mathbf{h}$ Western blot analysis of PGC1 $\alpha$ expression of the heart from sham and CKD mice. Microarray data were obtained from three mice per group. Data in $\mathbf{a}, \mathbf{b}$ are shown as - Log $P$ and were analyzed by Fisher's exact test, adjusted by using Benjamini-Hochberg. Data in e-h are shown as mean \pm SD and were analyzed by a two-tailed unpaired $t$-test $(n=3)$. ${ }^{\star} P<0.05,{ }^{\star \star} P<0.01,{ }^{\star \star \star} P<0.001$.

patients were divided into three groups according to tertiles of the serum phosphate level $(2.787-10.932 \mathrm{mg} / \mathrm{dl})$. We found that serum phosphate level was inversely associated with eGFR ( $r=$ $-0.4387, P<0.0001$ ) (Supplementary Fig. 2A). Since ejection fraction $(\mathrm{EF} \%)$ decreases and LVMI increases with decreasing eGFR, we used partial correlation analysis to analyze the associations of EF\% and LVMI with phosphate levels in the presence of adjusted eGFR. As shown in Supplementary Fig. 2B, $\mathrm{C}$, serum phosphate level was negatively associated with EF\% (the partial correlation coefficient $r_{\mathrm{p}}=-0.3442, P<0.0001$ ), while positively correlated with LVMI $\left(r_{\mathrm{p}}=0.3541, P<0.0001\right)$. Compared with participants in the lowest phosphate tertile, those in the highest tertile had the highest LVMI $(P=0.0001)$ (Supplementary Fig. 2B) and the lowest EF\% $(P=0.0004)$ (Supplementary Fig. 2C).
To evaluate the effect of HP on cardiac remodeling, H9c2 cells and NRVMs were treated with HP. We found that HP could induce cardiac hypertrophy, along with increased expressions of hypertrophic genes including atrial natriuretic factor (ANF), brain natriuretic peptide (BNP), and $\beta$-myosin heavy chain $(\beta-\mathrm{MHC})$ (Supplementary Fig. 3A, B). Meanwhile, we fed CKD mice with high phosphate diet (HPD) for 12 weeks and found that cardiac hypertrophy and failure were aggravated in HPD-fed CKD mice (Fig. 3a and Supplementary Fig. 3C-I). Next, we confirmed that the decrease in the expressions of PGC1a and its target genes was much more prominent in HPD-fed CKD mice than in CKD mice (Fig. 3b, c). Concordantly, more apparent changes in mitochondrial derangements, ATP level, and the expressions of FAO and OXPHO-related genes were observed in HPD-fed CKD mice (Fig. 3d-g). These data suggest that 
a

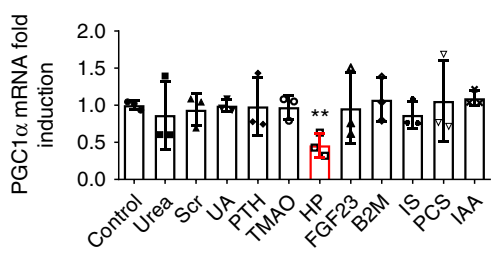

b

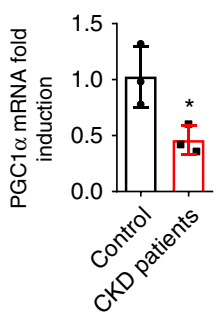

d

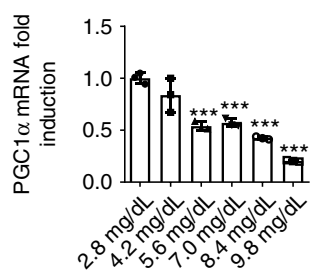

e

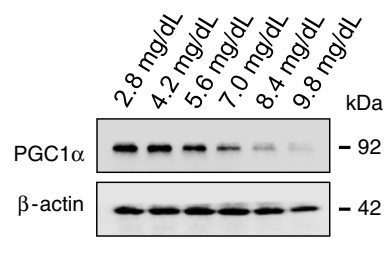

g
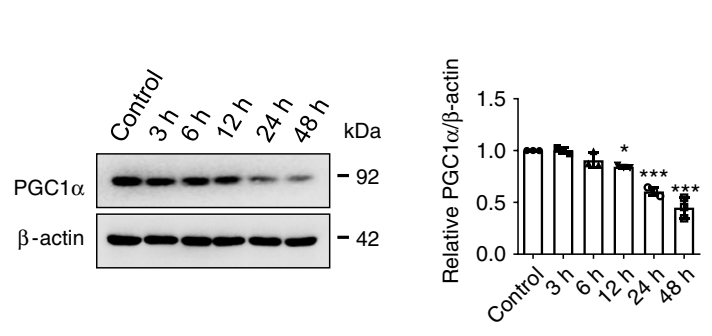

j
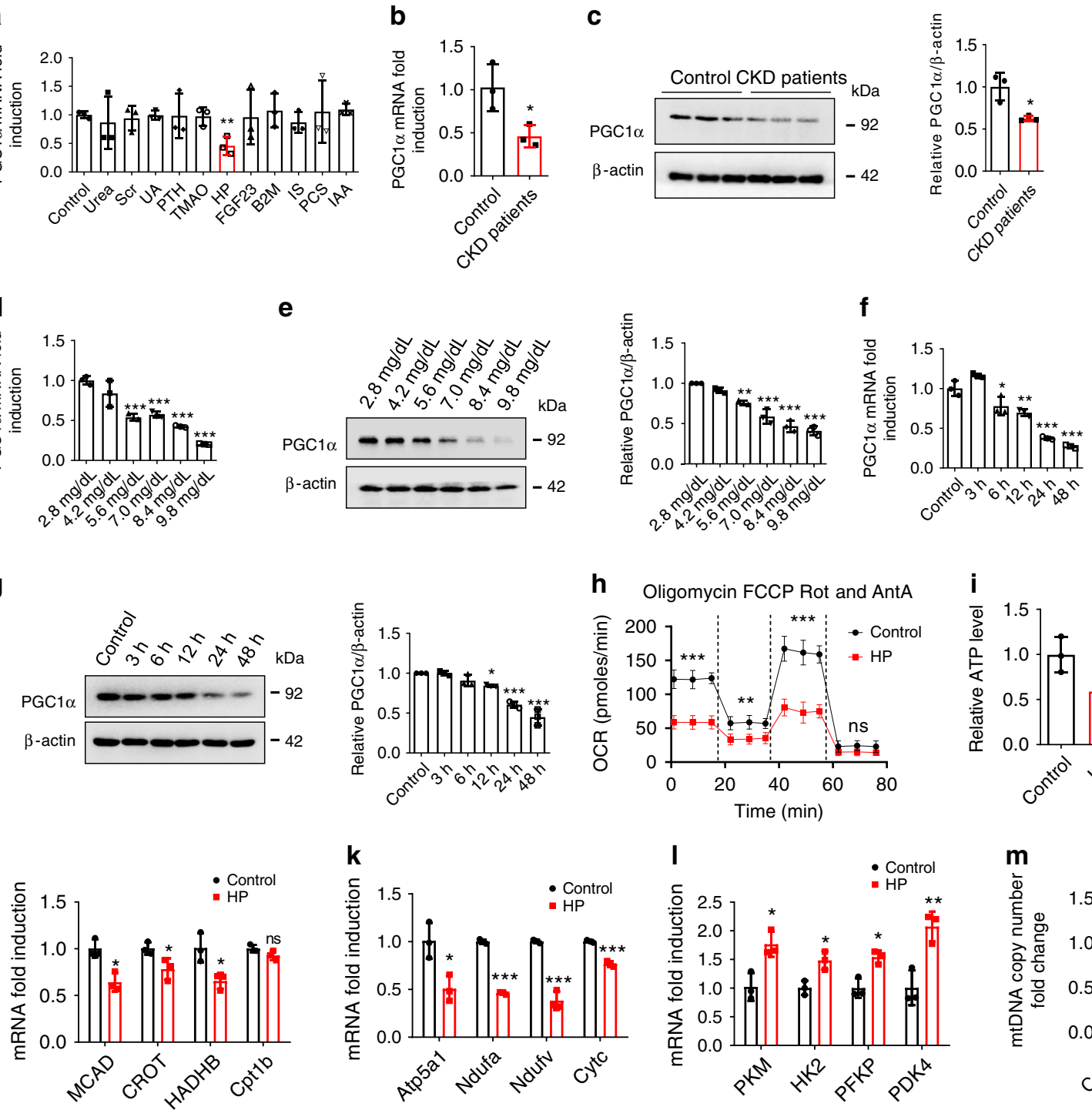

n
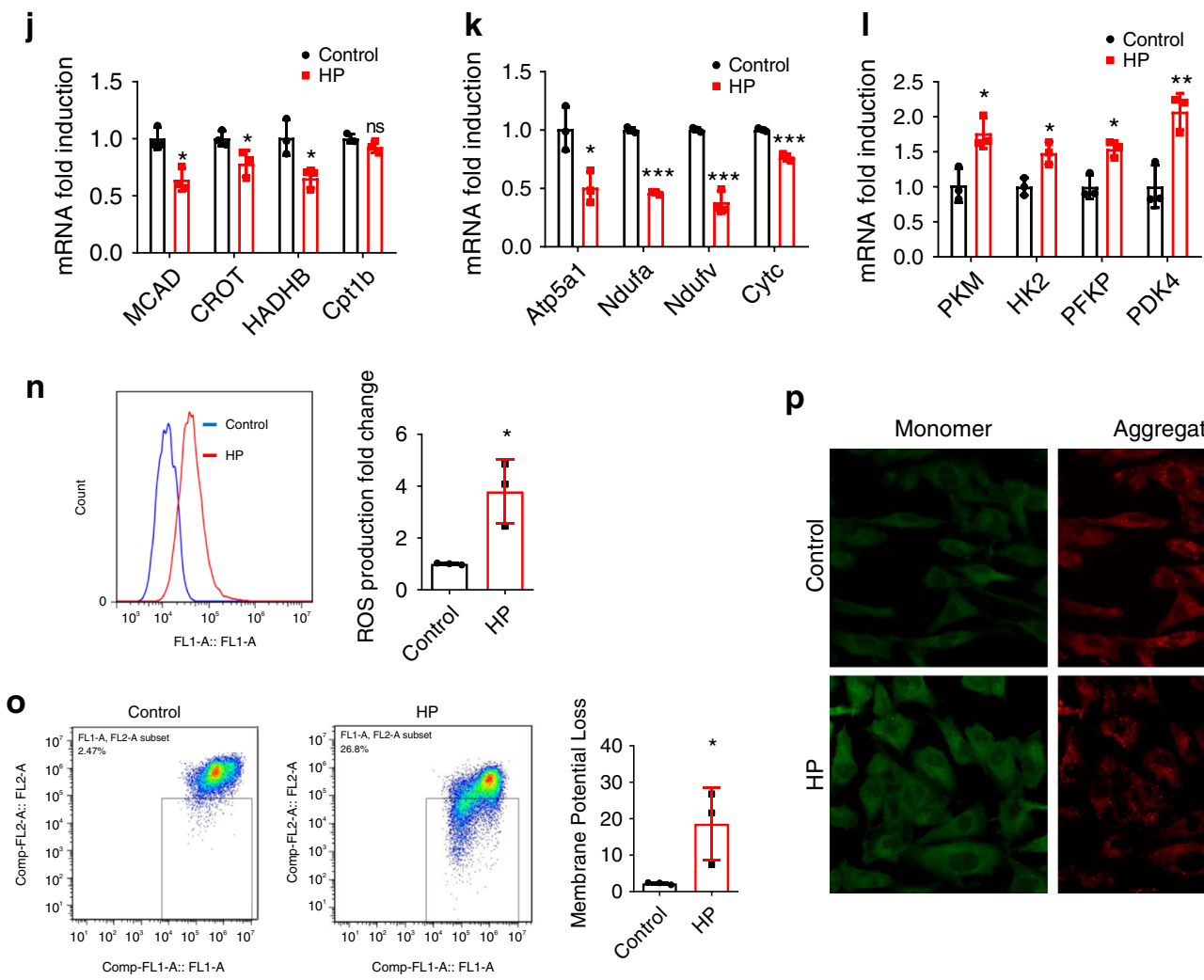

p
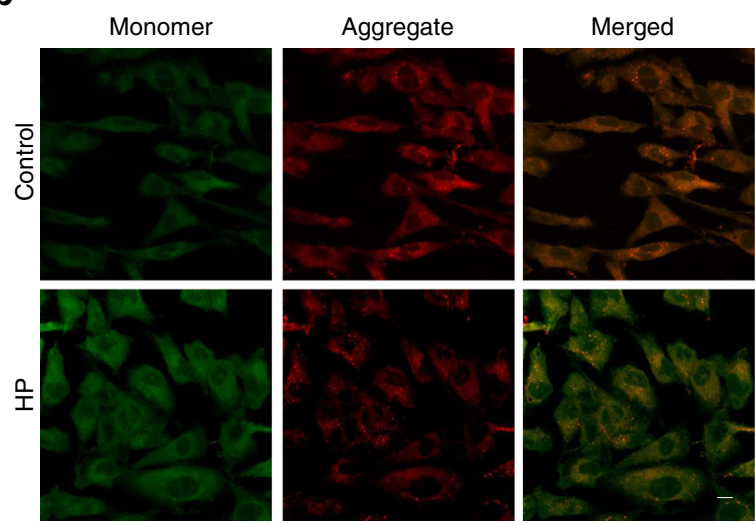

HP contributes to metabolic disorders and cardiac remodeling in vivo.

PGC1a protects against HP-induced pathological changes. As the preservation of PGC1 $\alpha$ expression has therapeutic potential for $\mathrm{HF}^{18,20}$, we sought to determine whether restoration of PGC1a expression could rescue HP-induced changes. As expected, both PGC1 $\alpha$ overexpression and nicotinamide (Nam, a key effector of PGC1 $\alpha^{17,21}$ ) treatment rescued $\mathrm{HP}$-induced disturbance of PGC1a expression, ROS production, metabolic 
Fig. 2 HP induces PGC1 $\alpha$ reduction and mitochondrial energy metabolism dysfunction in vitro. a H9c2 cells were treated with urea (1.2 mg/ml), serum creatinine $(\mathrm{Scr}, 80 \mu \mathrm{g} / \mathrm{ml})$, uric acid $(\mathrm{UA}, 80 \mu \mathrm{g} / \mathrm{ml})$, parathyroid hormone (PTH, $90 \mathrm{ng} / \mathrm{ml})$, trimethylamine- $\mathrm{N}$-oxide (TMAO, $0.751 \mu \mathrm{g} / \mathrm{ml}), \mathrm{HP}(84 \mu \mathrm{g} /$ $\mathrm{ml})$, FGF23 $(20 \mathrm{ng} / \mathrm{ml}), \beta 2$-microglobulin (B2M, $20 \mu \mathrm{g} / \mathrm{ml})$, indoxyl sulfate $(\mathrm{IS}, 125 \mu \mathrm{g} / \mathrm{ml})$, p-cresyl sulfate (PCS, $47 \mu \mathrm{g} / \mathrm{ml})$, and indole-3-acetic acid $(I A A, 3.5 \mu \mathrm{g} / \mathrm{ml}$ ), separately. mRNA was extracted for qPCR analysis of PGC1 $\alpha$ expression. b, c qPCR and western blot analysis of PGC1 $\alpha$ expression in $\mathrm{H} 9 \mathrm{c} 2$ cells incubated with the serum of healthy donors or CKD patients for $24 \mathrm{~h}$. $\mathbf{d}-\mathbf{g}$ qPCR and representative western blot analysis of PGC1 $\alpha$ expression in $\mathrm{H} 9 \mathrm{c} 2$ cells treated with control, various doses of HP for $24 \mathrm{~h}$ or HP $(8.4 \mathrm{mg} / \mathrm{dl})$ for various durations. h-p Oxygen consumption rate (OCR) (h), relative ATP level (i), relative mRNA expression of FAO genes $(\mathbf{j})$, relative mRNA expression of OXPHO genes ( $\mathbf{k})$, relative mRNA expression of glycolysis genes (I), relative mitochondrial DNA copy number $(\mathbf{m})$, ROS production $(\mathbf{n})$, and monomer and aggregate JC-1 (o flow cytometry; p representative images of laser scanning confocal microscopy. Scale bar, $10 \mu \mathrm{m}$ ) in $\mathrm{H} 9 \mathrm{c} 2$ cells treated with control or HP for $24 \mathrm{~h}$. Data are shown as mean \pm SD and were analyzed by a two-tailed unpaired $t$-test $(\mathbf{a}-\mathbf{c}, \mathbf{h}-\mathbf{o})$ or one-way ANOVA $(\mathbf{d}-\mathbf{g}) . n=3(\mathbf{a}-\mathbf{g}, \mathbf{i}-\mathbf{p})$ or $n=4(\mathbf{h})$ biologically independent experiments. ${ }^{\star} P<0.05,{ }^{\star \star} P<$ $0.01,{ }^{\star \star \star} P<0.001$.
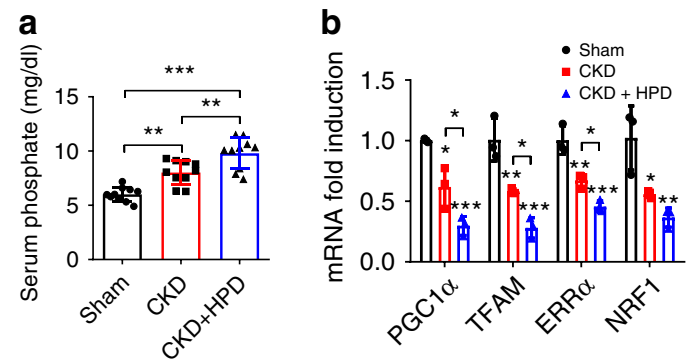

C

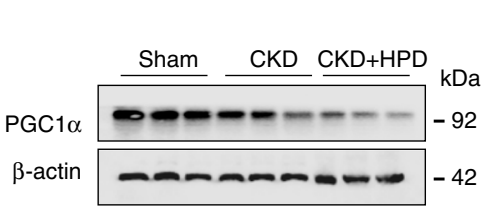

d

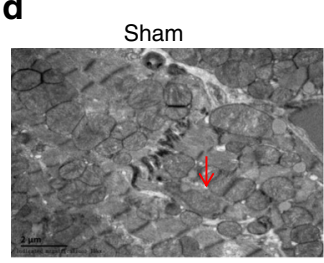

f

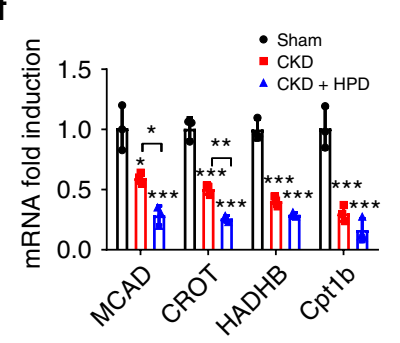

CKD

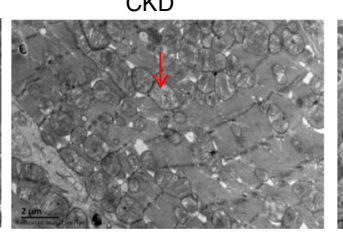

CKD+HPD

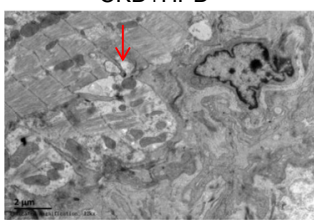

g

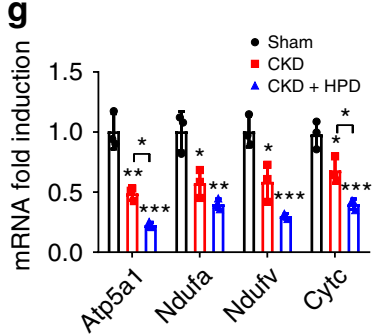

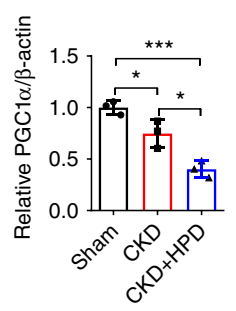

e

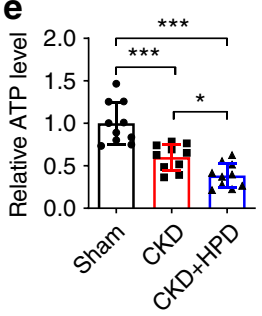

Fig. 3 HP induces PGC1 $\alpha$ suppression and cardiac remodeling in CKD mice. a Serum phosphate level in sham, CKD, and HPD-fed CKD mice for 12 weeks. $n=10$ mice per group. b-g qPCR analysis of PGC1 $\alpha$ and its target genes expressions (b), western blot analysis of PGC1 $\alpha$ expression (c), representative images of TEM observation of mitochondria (d). Red arrows point to mitochondria, relative ATP level (e), relative mRNA expression of FAO genes (f), and relative mRNA expression of OXPHO genes $(\mathbf{g})$ in heart tissues from the mice in $\mathbf{a} . n=10(\mathbf{a}, \mathbf{e})$, or $n=3$ (b-d, $\mathbf{f}, \mathbf{g})$. Data are shown as mean \pm SD and were analyzed by one-way ANOVA. ${ }^{\star} P<0.05,{ }^{\star \star} P<0.01,{ }^{\star \star \star} P<0.001$.

genes expression, ATP level, and cardiomyocyte hypertrophy (Fig. 4a-d and Supplementary Fig. 4A). Concordantly, cardiac hypertrophy, heart failure, and myocardial energy metabolism remodeling in HPD-fed CKD mice were significantly ameliorated by Nam treatment (Fig. $4 \mathrm{e}-\mathrm{j}$ and Supplementary Fig. 4B-D).

HP regulates PGC1a expression and energy metabolism via IRF1. Then, we tried to explore the mechanism underlying HPinduced PGC1 1 downregulation. Bioinformatics analysis predicted 36 transcriptional factors that could bind to PGC1a promoter region, 8 of which [IRF1, zinc finger protein 354C (ZNF354C), Meishomeobox 1 (MEIS1), transcription factor 12 (Tcf12), forkhead box L1 (FOXL1), poly(A) binding protein cytoplasmic 1 (PABPC1), breast cancer type 1 susceptibility protein (BRCA1), and insulin-like growth factor 1 receptor
(IGF1R)] were associated with cardiac hypertrophy. Interestingly, IRF1 was the only upregulated transcriptional factor in HPtreated cells (Fig. 5a-c). Moreover, the expression level of IRF1 was much higher in H9c2 cells than that of other IRF family members unable to be regulated by HP (Supplementary Fig. 5A, B). Of note, a more remarkable increase of IRF1 expression was observed in HPD-fed CKD mice than in control or CKD mice (Fig. 5d, e). Furthermore, IRF1 expression was also upregulated in H9c2 cells incubated with CKD patients' serum (Fig. 5f, g) and in NRVMs after HP treatment (Supplementary Fig. 5C, D). Considering the crucial role of IRF1 in cardiac hypertrophy ${ }^{22}$, we hypothesized that HP might suppress PGCla expression via upregulating IRF1 expression. To test this hypothesis, two pairs of different siRNAs against IRF1 (siIRF1) were transfected into H9c2 cells, and we found that both of them could rescue the HPinduced disturbance of PGC1a expression, ROS production, ATP level, metabolic gene expression and cardiomyocyte hypertrophy 

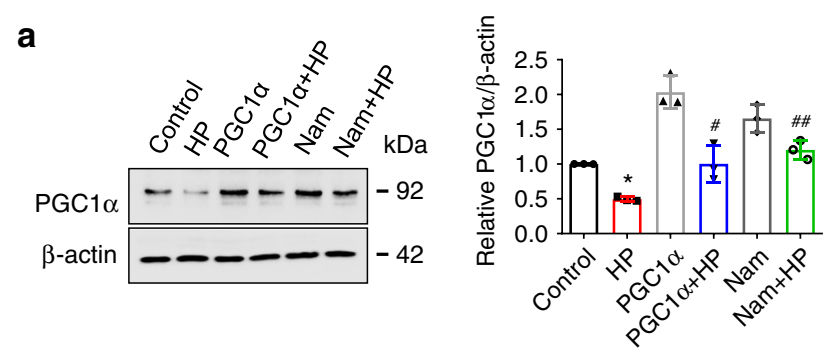

C
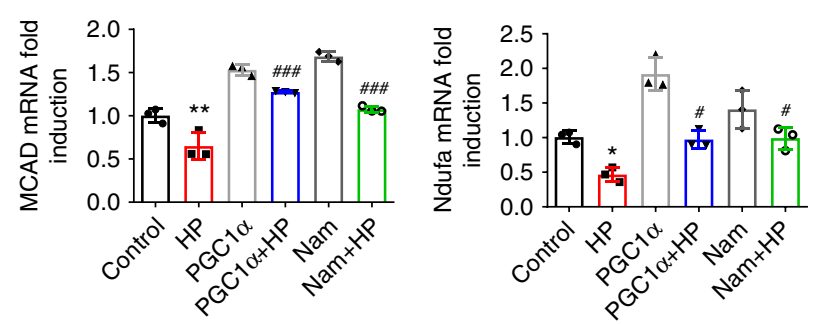

e
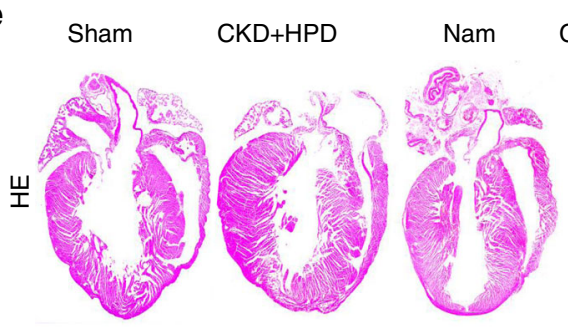

$\mathrm{CKD}+\mathrm{HPD}+\mathrm{Nam}$
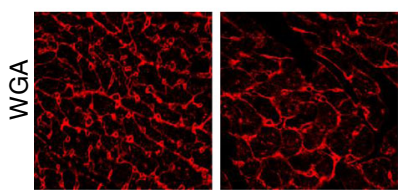
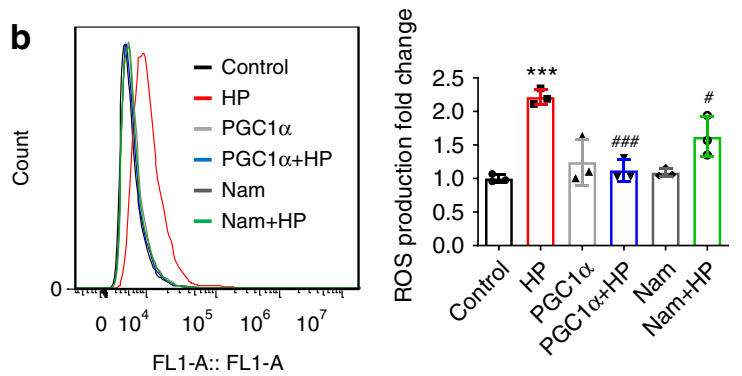

d
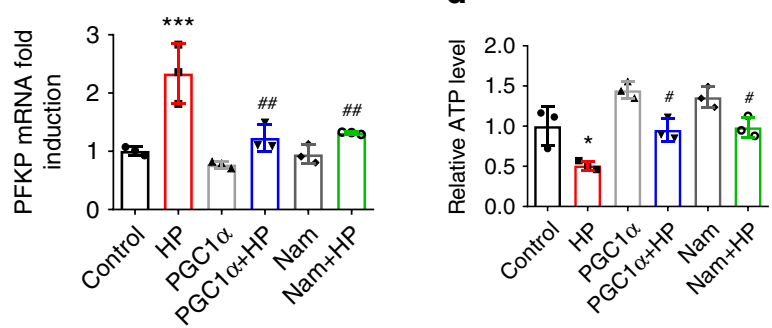

h
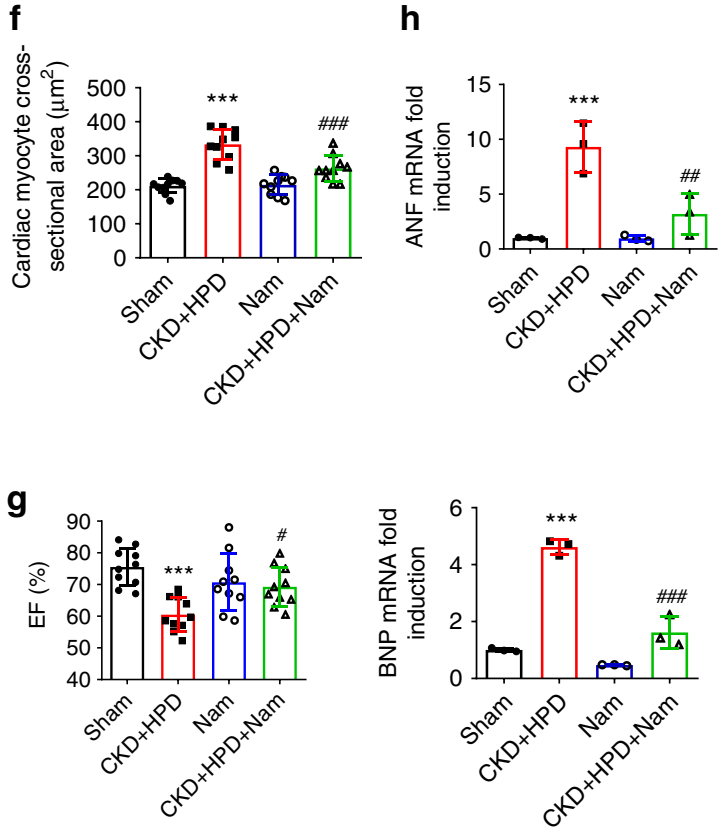

i
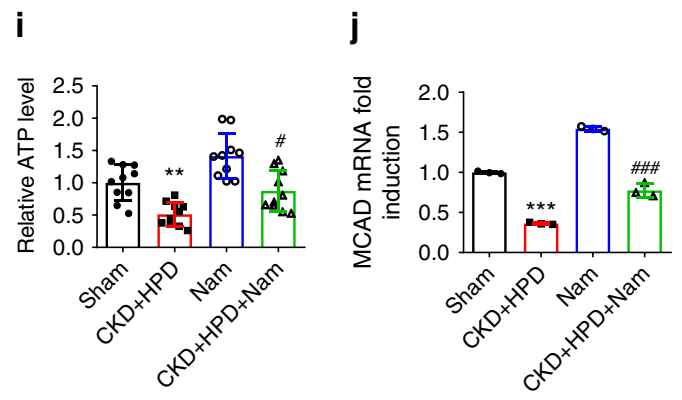

(Fig. 5h-l and Supplementary Fig. 5E, F). Moreover, PGC1a was downregulated in IRF1 overexpression plasmids-transfected cells (Fig. $5 \mathrm{~m}, \mathrm{n}$ ), and increased expressions of PGC1 $\alpha$, PGC1 $\alpha$ target genes, and FAO- and OXPHO-related genes were detected in IRF1 ${ }^{-1-}$ mice (Supplementary Fig. 5G-K). All these data support the hypothesis that IRF1 may mediate the effect of HP on PGC1a downregulation.
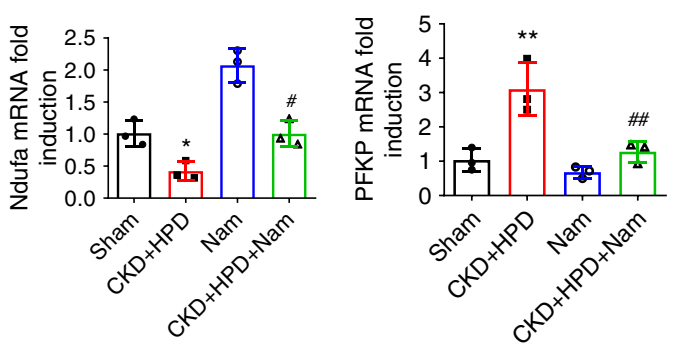

IRF1 directly binds to PGC1a promoter region for inhibition. Next, we explored the mechanism by which IRF1 regulates PGC1a expression. We first found that the mRNA stability and protein degradation of PGCla were not significantly affected by HP (Supplementary Fig. 6). Of note, bioinformatics analysis showed that IRF1 had the potential to bind to the PGC1a promoter region (Supplementary Table 1), hinting that IRF1 may 
Fig. 4 Restoration of PGC1 $\alpha$ expression protects against HP-induced mitochondrial energy metabolism dysfunction and cardiac remodeling in both cardiac cells and CKD mice. a-d H9c2 cells were transfected with PGC1 $\alpha$ overexpression plasmids or pretreated with Nam, and then treated with control or HP for representative western blot analysis of PGC1 $\alpha$ expression (a) ROS production (b) relative mRNA expression of MCAD (FAO), Ndufa (OXPHO), and PFKP (glycolysis) (c), and relative ATP level (d). e Representative gross pathology (HE staining, upper panel. Scale bar, $1 \mathrm{~mm}$ ) and WGA staining (lower panel. Scale bar, $10 \mu \mathrm{m}$ ) of heart sections from sham, HPD-fed CKD, Nam, and HPD-fed CKD mice intraperitoneally injected with Nam for 12 weeks. $n=10$ mice per group. $\mathbf{f}$ Cross-sectional surface area of individual cardiac myocytes from the mice in the lower panel of (e). $\mathbf{g}$-j Echocardiography of EF\% $(\mathbf{g})$, QPCR analysis of the mRNA expression of hypertrophic genes $(\mathbf{h})$, relative ATP level (i), relative mRNA expression of metabolic genes (j) of heart lysates from the mice in $(\mathbf{e}) . n=3(\mathbf{a}-\mathbf{d}, \mathbf{h}, \mathbf{j})$, or $n=10(\mathbf{e}-\mathbf{g}, \mathbf{i})$. Data are shown as mean \pm SD and were analyzed by one-way ANOVA. ${ }^{\star} P<0.05,{ }^{\star \star} P<$ $0.01,{ }^{\star \star \star} P<0.001$ versus control or sham. ${ }^{\#} P<0.05, \# \# P<0.01, \# \# \#<0.001$ versus HP or CKD + HPD.

inhibit PGC1a expression via directly binding to its promoter region. To prove this speculation, six truncated PGC1a reporter plasmids were transfected into H9c2 cells. As shown in Fig. 6a, HP markedly inhibited the luciferase activities of the reporter plasmids pGL3-PGC1 $\alpha$-1703, pGL3-PGC1 $\alpha$-1524, pGL3-PGC1 $\alpha$ 985 and pGL3-PGC1 -719 (rather than pGL3-PGC1 $\alpha-348$ or pGL3-PGC1 $\alpha-183$ ), indicating an IRF1 response element within the sequence containing -719 to -348 nucleotides relative to the transcriptional start site. Therefore, the putative binding sequence should be the portion containing -632 to -612 nucleotides (TCCTTCTTTCTTTTCCCTATT) according to bioinformatics analysis (Supplementary Table 1). We then demonstrated that mutation of the IRF1 response element in this putative binding sequence ( -632 to -612 ) almost abolished HP-regulated PGC1a promoter activity (Fig. 6b), while a mutation in the sequence containing -974 to -954 nucleotides was unable to rescue HPmediated changes (Supplementary Fig. 7). ClustalW analysis showed that the sequence containing -632 to -612 nucleotides in the PGC1a promoter region was highly conserved among 8 different mammals (Supplementary Table 2). To determine whether IRF1 can bind to the PGC1a promoter region directly, chromatin immunoprecipitation (ChIP) assay was performed. The assay revealed direct binding of IRF1 to the PGC1 a promoter region $(-709$ to -511 ), which could be enhanced by HP (Fig. 6c, d), compared with a negative control.

Subsequently, we attempted to gain mechanistic insight into the regulation of IRF1 expression by HP. To investigate how phosphate is transported into cardiomyocytes, we screened the expressions of phosphate transporters and found that solute carrier family 20 member 1 (SLC20A1, Pit1) and SLC20A2 (Pit2) were highly expressed in both $\mathrm{H} 9 \mathrm{c} 2$ cells and NRVMs (Supplementary Fig. 8A). We then revealed that Pit1 and Pit2 could be upregulated by HP (Fig. 6e), while simultaneous knockdown of Pit1 and Pit2 could evidently decrease intracellular phosphate level and abrogate HP-induced IRF1 upregulation, PGC1 $\alpha$ downregulation and cardiomyocyte hypertrophy (Fig. 6e, $\mathrm{f}$ and Supplementary Fig. 8B-E), indicating that the action of HP depends on the transporters Pit1 and Pit2.

To further uncover the mechanisms underlying the regulation of IRF1 expression by HP, we examined the contributions of DNA methylation and histone acetylation, as HP has the potential to regulate DNA methylation and histone acetylation ${ }^{23}$. Methylationspecific PCR (MSP) using two different primers showed no significant change of DNA methylation in the IRF1 promoter region after HP treatment (Supplementary Fig. 9A, B). However, more acetylated H3K9 (Fig. 6g, h), rather than H4K12 (Supplementary Fig. 10), was found to bind to the IRF1 promoter region after HP treatment, suggesting that histone $\mathrm{H} 3 \mathrm{~K} 9$ acetylation occurred. We then demonstrated that HP-induced expressions of histone acetyltransferases [E1A binding protein p300 (P300), CREB binding protein (CBP), and histone acetyltransferase 1 (HAT1)] (Fig. 6i). The inhibition of $\mathrm{H} 3 \mathrm{~K} 9$ acetylation by P300/CBP inhibitor (C646) or pan-HAT inhibitor (anacardic acid, AA) significantly attenuated HP-induced IRF1 upregulation and PGC1a downregulation (Fig. 6j-1). These data further validate the role of $\mathrm{H} 3 \mathrm{~K} 9$ acetylation in HP-regulated IRF1 transcription.

$\boldsymbol{k l} / \boldsymbol{k l}$ mice display similar changes to HP-induced effects. Given the serological characteristics of hyperphosphatemia that accompanied CKD and CVD in Klotho mutant $(\mathrm{kl} / \mathrm{kl}) \mathrm{mice}^{24,25}$, we then wondered whether the HP-induced changes in energy metabolism remodeling could also be detected in cardiomyocytes in $\mathrm{kl} / \mathrm{kl}$ mice. As anticipated, the serum phosphate level was significantly higher in $\mathrm{kl} / \mathrm{kl}$ mice $(12.72 \pm 1.99 \mathrm{mg} / \mathrm{dl})$ than in wild type (WT) mice $(6.35 \pm 0.86 \mathrm{mg} / \mathrm{dl})$ (Fig. $7 \mathrm{a})$, even higher than in HPD-fed CKD mice $(9.79 \pm 1.50 \mathrm{mg} / \mathrm{dl})$ (Fig. 3a). Notably, cardiac hypertrophy and failure were also observed in $\mathrm{kl} / \mathrm{kl}$ mice (Fig. 7b-g), accompanied by decreased PGCla expression, increased IRF1 expression, mitochondrial dysfunction, and energy metabolism remodeling (Fig. $7 \mathrm{~h}-\mathrm{m}$ ). In addition, a higher serum phosphate level along with dysregulated IRF1-PGC1 $\alpha$ axis and energy metabolism remodeling were observed in adenineinduced CKD mice (Supplementary Fig. 11A-K), further confirming the role of hyperphosphatemia in CKD-associated $\mathrm{HF}$ and involved mechanism.

Studies have shown that mutual interactions exist among klotho, FGF23, PTH, and Vitamin D (VitD) during their regulation of phosphate levels in vivo ${ }^{24,25}$. Since hyperphosphatemia and high levels of FGF23 were both detected in $k l / k l, C K D$, and HPD-fed mice (Supplementary Fig. 12A-C), we then explored the effect of FGF23 on HP-mediated changes. Interestingly, an additive effect between FGF23 and HP was observed in inducing cardiac hypertrophy and energy metabolism remodeling (Supplementary Fig. 13A-D). However, inhibitors of neither FGF23 nor FGF23 receptor (FGFR) had effects on the expressions of IRF1 and PGC1a (Supplementary Fig. 13E-O), reflecting that HP-mediated alterations in the IRF1-PGC1a axis and the subsequent energy metabolism remodeling are independent of FGF23.

Genetic knockdown of IRF1 protects against HPD-induced HF. Finally, we verified the role of IRF1 in HP-induced changes in vivo. As shown in Fig. 8, cardiac hypertrophy and failure, PGC1a downregulation, and energy metabolism remodeling in HPD-fed CKD mice were significantly ameliorated by knocking down IRF1 expression in $\mathrm{IRF}^{+/-}$mice (Fig. 8a-h). To better clarify the direct role of IRF1 in HP-induced alterations, we fed IRF1 knockout (IRF1 $1^{-/}$) mice with HPD for 12 weeks and found that HPD-induced cardiac hypertrophy, HF, downregulated PGC1 $\alpha$ expression and energy metabolism remodeling were significantly attenuated in IRF $1^{-1-}$ mice (Supplementary Fig. 14A-J). These results demonstrate that IRF1 not only has a distinctive role in HP-induced $\mathrm{HF}$, but also is a potential target for the treatment of CKD-associated CVD.

\section{Discussion}

CRS4, a prevalent complication of CKD with a high incidence, has emerged as a leading cause of death in CKD patients ${ }^{1,2,5}$, but the pathogenic mechanism remains elusive. In the present study, 

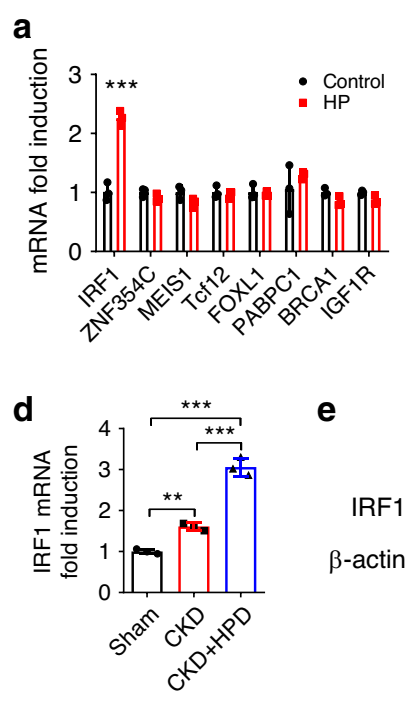

g
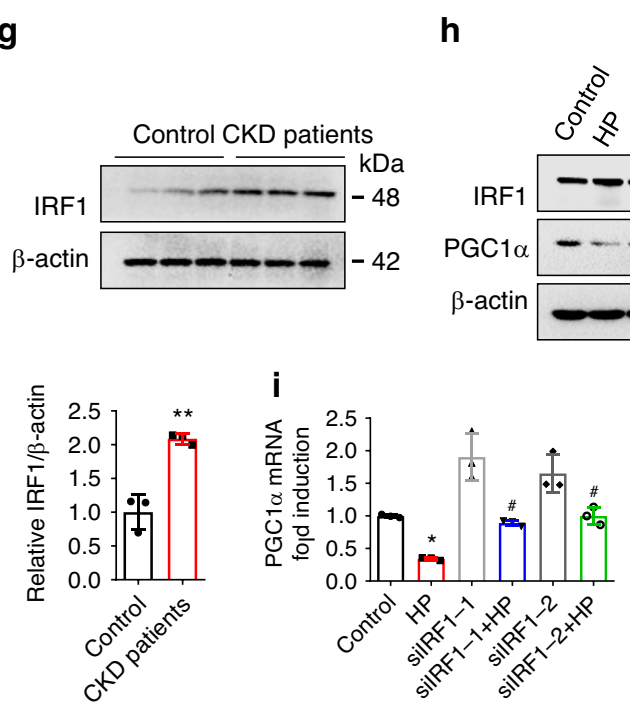
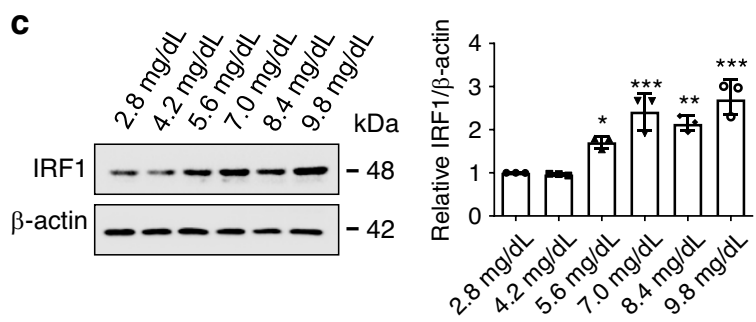
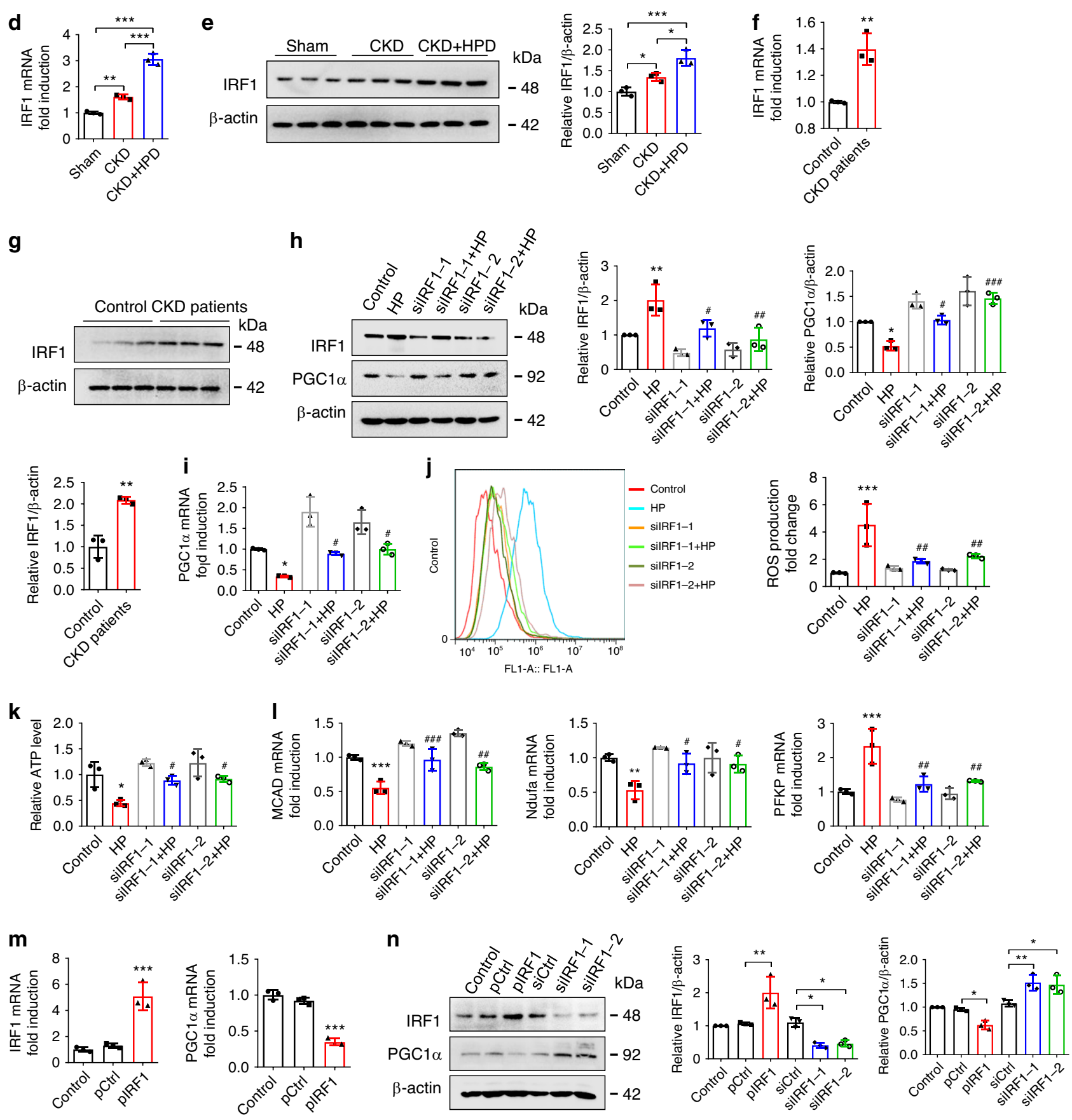

we demonstrate that PGC1 $\alpha$ downregulation and consequent myocardial energy metabolism remodeling contribute to CKDassociated HF, during which hyperphosphatemia has a pivotal role. Mechanistically, HP disturbs PGC1a expression through epigenetic regulation of IRF1. Pharmacological restoration of
PGC1 $\alpha$ expression or genetic knockdown of IRF1 protects against CKD-associated myocardial energy metabolism remodeling and HF (Fig. 9).

Multiple risk factors contribute to HF, including hypertension, hemodynamic alterations, RAAS activation, and sympathetic 
Fig. 5 HP disturbs PGC1 $\alpha$ expression and energy metabolism in an IRF1-dependent manner. a qPCR analysis of the expression of the predicted transcriptional factors in $\mathrm{H} 9 \mathrm{c} 2$ cells treated with control or HP for $24 \mathrm{~h} . \mathbf{b}, \mathbf{c}$ qPCR and representative western blot analysis of IRF1 expression in H9c2 cells treated with control or various doses of HP for $24 \mathrm{~h}$. $\mathbf{d}-\mathbf{g}$ qPCR and representative western blot analysis of IRF1 expression in heart lysates from sham, CKD, and HPD-fed CKD mice for 12 weeks (d, e) or in H9c2 cells incubated with the serum of healthy donors or CKD patients for $24 \mathrm{~h}(\mathbf{f}, \mathbf{g})$. h-I H9c2 cells were transfected with two pairs of siRNA against IRF1, and then treated with control or HP for $24 \mathrm{~h}$. Cells were collected for detection of IRF1 and PGC1 $\alpha$ protein expressions (h), PGC1 $\alpha$ mRNA expression (i), ROS production (j), relative ATP level ( $\mathbf{k})$, and mRNA expression of the metabolic genes (I). $\mathbf{m}$ qPCR analysis of IRF1 and PGC1 $\alpha$ expressions in H9c2 cells transfected with control plasmids ( $p$ Ctrl) or IRF1 overexpression plasmids (pIRF1). $\mathbf{n}$ Representative western blot analysis of IRF1 and PGC1 $\alpha$ expressions in H9c2 cells transfected with pCtrl, pIRF1, control siRNA (siCtrl), and two pairs of siRNA against IRF1 (silRF1), separately. $n=3$ biologically independent experiments (a-n). Data are shown as mean \pm SD and were analyzed by a two-tailed unpaired $t$-test (a, $\mathbf{f}, \mathbf{g}$ ) or one-way ANOVA (b-e, h-n). ${ }^{\star} P<0.05,{ }^{\star \star} P<0.01,{ }^{\star \star \star} P<0.001$ versus control. ${ }^{*} P<0.05, \# \# P<0.01, \# \# \#<0.001$ versus HP.

a

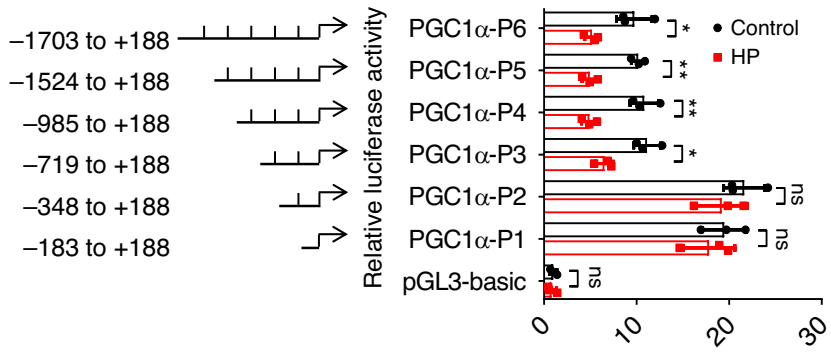

b
C

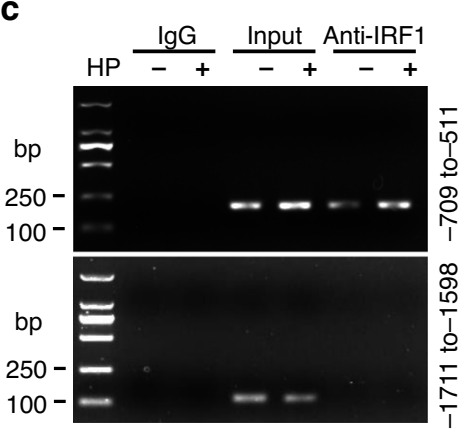

d

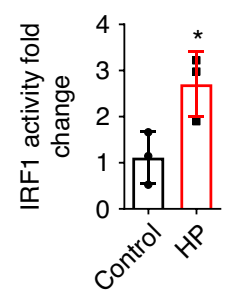

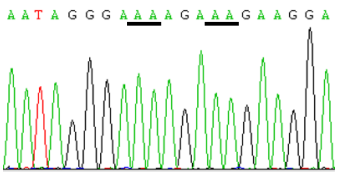

pGL3-PGC1 $\alpha-M 3$

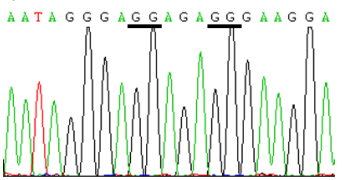

pGL3-PGC1 $\alpha-P 3$

- Control

- HP

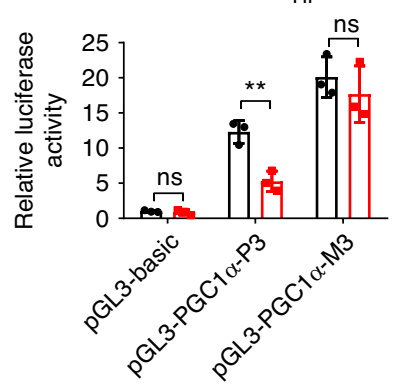

e

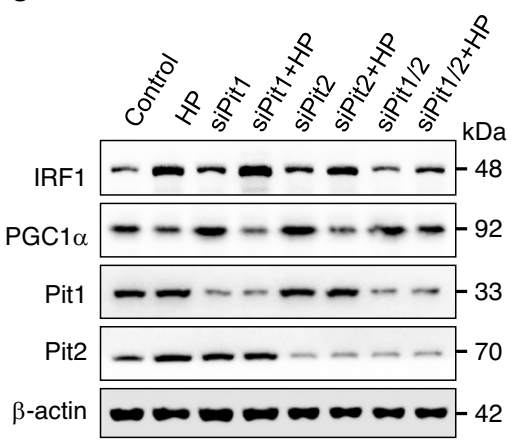

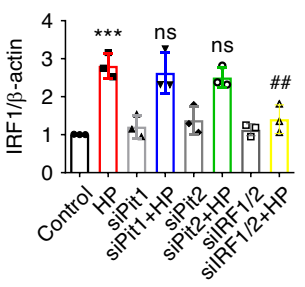

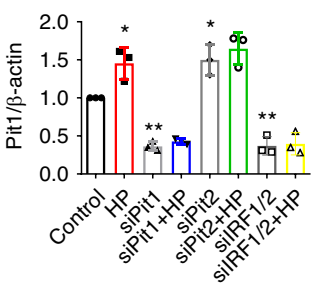

f

i

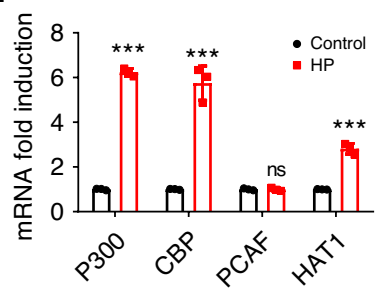

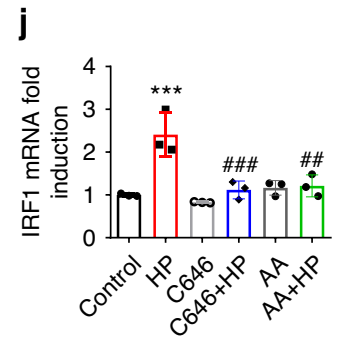
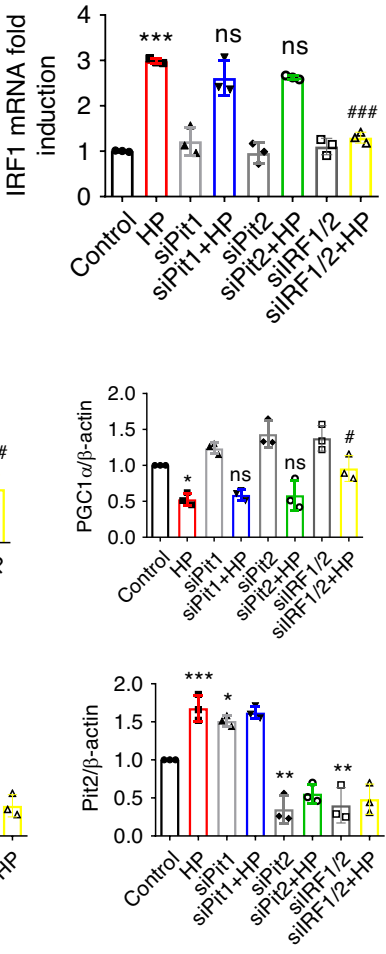

k

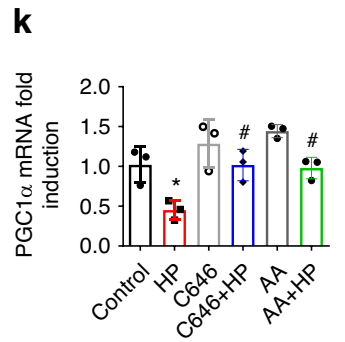

I
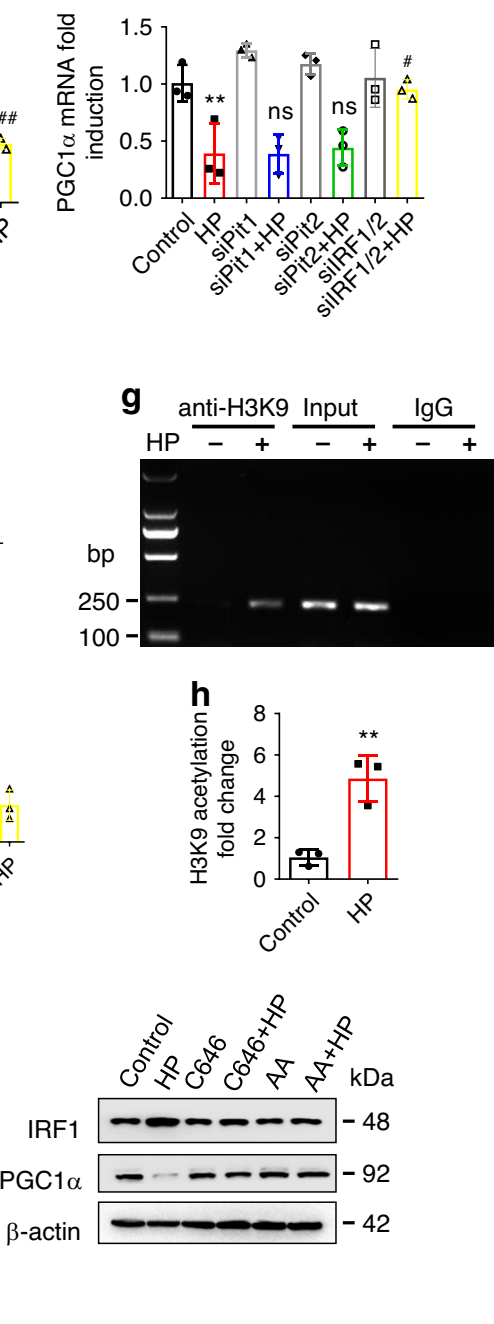
Fig. 6 IRF1 inhibits PGC1 $\alpha$ expression via directly binding to its promoter region. a After being co-transfected with pRL-TK vector and pGL3-basic or recombinant reporter plasmids containing various fragments of $\mathrm{PGC1} 1 \alpha$ promoter region, $\mathrm{H} 9 \mathrm{c} 2$ cells were treated with control or HP and then harvested for dual-luciferase reporter assay. The firefly luciferase activity was normalized against Renilla activity. $\mathbf{b} \mathrm{H} 9 \mathrm{c} 2$ cells were co-transfected with pRL-TK vector and pGL3-PGC1 $\alpha-P 3$ or pGL3-PGC1 $\alpha-M 3$ (containing the mutant bases of pGL3-PGC1 $\alpha-P 3$ underlined in the sequencing results), treated with control or HP and harvested for luciferase assay. c, $\mathbf{d}$ H9c2 cells were treated with control or HP for $24 \mathrm{~h}$ and harvested for ChIP assay. IRF1 antibody was immunoprecipitated with chromatin DNA fragments, taking IgG as a negative control. The precipitated DNA was amplified by PCR $\mathbf{c}$ and qPCR $\mathbf{d}$ using primers that cover the PGC1 $\alpha$ promoter region $(-709$ to -511$)$, taking primers that cover the region ( -1711 to -1598$)$ as a negative control. e, f $\mathrm{qPCR}$ and representative western blot analysis of IRF1 and PGC1 $\alpha$ expressions in cells treated with siPit1, siPit2, or both in the presence or absence of HP. $\mathbf{g}$, $\mathbf{h} \mathrm{H} 9 \mathrm{c} 2$ cells were treated with control or HP, and H3K9 acetylation was assayed using ChIP. i qPCR analysis of histone acetyltransferase genes expression in H9c2 cells treated with control or HP. $\mathbf{j}-\mathbf{I}$ qPCR and western blot analysis of IRF1 and PGC1 $\alpha$ expressions in cells treated with a P300/CBP inhibitor (C646, $10 \mu \mathrm{M}$ ) or a pan-histone acetyltransferase inhibitor (anacardic acid, AA,10 $\mu \mathrm{M}$ ) in the presence or absence of HP. Data are shown as mean \pm SD and were analyzed by a two-tailed unpaired $t$-test $(\mathbf{a}-\mathbf{d}, \mathbf{h}, \mathbf{i})$ or one-way ANOVA $(\mathbf{e}, \mathbf{f}, \mathbf{j}, \mathbf{k}) . n=3$ biologically independent experiments (a, $\mathbf{b}, \mathbf{d}-\mathbf{f}, \mathbf{h}-\mathbf{k})$. ${ }^{\star} P<0.05,{ }^{\star \star} P<0.01,{ }^{\star \star \star} P<<0.001$ versus control. ${ }^{*} P<0.05,{ }^{*} P<0.01, \# \# P<0.001$, ns: no significance versus HP.

nervous system activation, but they are insufficient to explain the high prevalence of HF in CKD patients ${ }^{10,11}$. In this study, microarray analysis and TEM observation identified both functional and morphological changes of mitochondria in myocardial tissues of CKD mice. As reported, HF mostly occurs in CKD patients at stages $4-5$, especially in dialysis patients ${ }^{2,26}$. As the accumulation of uremic toxins is the most prominent and specific feature of CKD patients ${ }^{2,26}$, we screened CKD-specific toxins and identified $\mathrm{HP}$ as the toxin able to induce energy metabolism dysfunction. As known, phosphorus is an essential element for energy production and transfer ${ }^{27}$. However, long-term accumulation of phosphate is detrimental to health ${ }^{27}$. As reported in a community-based study enrolling 4494 participants without CVD, each $20 \%$ increase in dietary phosphate intake was associated with an estimated $1.1 \mathrm{~g}$ greater left ventricular mass $(\mathrm{LVM})^{28}$. In contrast, phosphate binders can prevent aortic stiffness, diastolic dysfunction, and LVH in CKD mice ${ }^{29}$, and are also able to improve the survival rate in CKD patients ${ }^{30}$. However, the mechanisms underlying the harmful effects of hyperphosphatemia remain unclear $^{12}$.

It is known that the functional and morphological changes of mitochondria affect ATP production in cardiomyocytes, thereby disrupting cardiac function and eventually leading to $\mathrm{HF}^{6-8}$. In the present study, we found that HP treatment led to mitochondrial energy metabolism remodeling via inhibiting the expression of PGC1a, a master regulator of mitochondrial biogenesis and energy metabolism ${ }^{17,18,20}$. Growing evidence suggests that downregulation of PGC1a may initiate the switch from fatty acid oxidation to glucose utilization ${ }^{18,31}$, especially in the process of chronic heart disease ${ }^{31,32}$. In this study, to our best knowledge, we are the first to discover that HP suppressed PGC1 $\alpha$ expression both in vitro and in vivo. We also found that PGC1a suppression induced dysregulation of FAO-associated genes possibly through affecting NRF1, a target gene of PGC1a, and an important regulator of OXPHO and $\mathrm{FAO}^{33,34}$ (Supplementary Fig. 15A-D). Interestingly, although many FAO-related genes are controlled by peroxisome proliferator-activated receptor $\alpha$ (PPAR $\alpha$ ), of which PGC1 $\alpha$ is a coactivator, we found no significant change in PPAR $\alpha$ expression after HP treatment (Supplementary Fig. 15E, F), further reflecting that HP-induced dysregulation of FAO-related genes is due to PGCla downregulation. On the other hand, the restoration of PGC1a expression significantly rescued HPinduced cardiomyocyte hypertrophy and energy metabolism dysfunction, suggesting that PGC1 $\alpha$ is a potential therapeutic target for CKD-associated $\mathrm{HF}^{18,20,31}$.

Normally, HP is unable to directly regulate PGC1a expression at the transcriptional level. By bioinformatics analysis, we revealed that IRF1 was responsible for the downregulation of PGC1a in HP-treated cardiomyocytes. IRF1 is known as an important regulator of genes involved in immune responses, inflammatory reactions, and tumor development ${ }^{35}$. IRF1 has a repressor domain, which contributes to its growth inhibitory activity $^{35}$. As reported, IRF1 can directly bind to the IRF response element in the promoter region of the target gene to negatively regulate its transcription ${ }^{36}$. Here, we found that PGC1a was a target gene of IRF1 and IRF1 suppressed PGC1 $\alpha$ expression via directly binding to its promoter region $(-632$ to -612$)$. This negative regulation of PGC1 $\alpha$ by IRF1 was further confirmed by the finding that knockdown of IRF1 significantly rescued HPinduced downregulation of PGC1 $\alpha$, mitochondrial energy metabolic dysfunction, and cardiac hypertrophy. Of note, IRF1 has been reported to be involved in CVDs by activating inducible nitric oxide synthase (iNOS) and affecting the interleukin-18 (IL18)-osteopontin (OPN) signaling pathway ${ }^{22,37}$. In this study, we demonstrate that the increase of IRF1 expression can induce energy metabolism remodeling by directly downregulating PGCla in cardiomyocytes, thus providing new insight into the mechanisms of IRF1-induced cardiac hypertrophy. In addition, although some other members of the IRFs family were reported to be associated with $\mathrm{HF}^{38}$, their expressions were not significantly changed after HP treatment, reflecting that IRF1 is a distinctive target of HP.

As known, HP is capable of inducing epigenetic modifications, including DNA methylation and histone acetylation ${ }^{23,39}$, which mediate the effects of HP, such as fibrotic fibroblast activation and osteogenic transdifferentiation ${ }^{39,40}$. As cardiac hypertrophy and HF could be rescued by inhibition of DNA methylation or histone acetylation ${ }^{41,42}$, methylation and acetylation may also have important roles in the pathogenesis of cardiac hypertrophy. However, the underlying mechanisms are not fully uncovered, and it is unknown whether HP can induce epigenetic modifications of IRF1. In this study, the effect of HP on the methylation of IRF1 was excluded, because no significant change of methylation in CPG islands of IRF1 promoter was detected after HP treatment. Instead, we detected acetylation of histone H3K9 in the IRF1 promoter region, and increased expression of HAT was observed after HP treatment. Moreover, pretreatment with HAT inhibitors could significantly attenuate HP-induced IRF1 upregulation and PGC1a downregulation. Therefore, HP-induced upregulation of IRF1 and subsequent downregulation of PGC1a are probably due to the ability of HP to induce histone acetylation.

Phosphate is transported into cells primarily through three transporter families: type I (SLC17 family), type II (SLC34 family), and type III (SLC20 family) transporters ${ }^{43}$. In this study, after screening we found that type III phosphate transporters, Pit1 and Pit2, were highly expressed in cardiomyocytes and also upregulated by HP. Interestingly, we found that a compensatory 
a

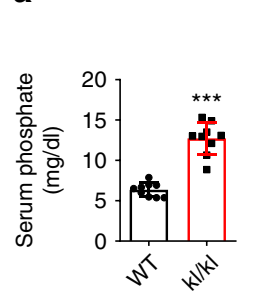

b

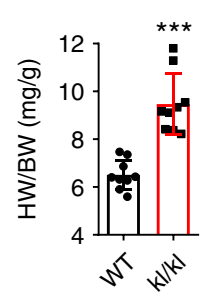

C

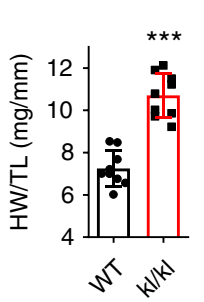

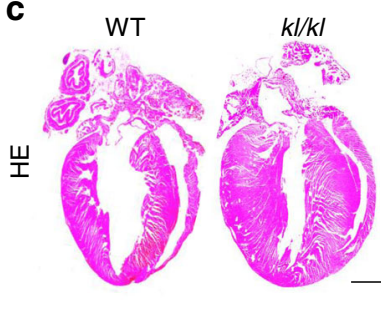

d

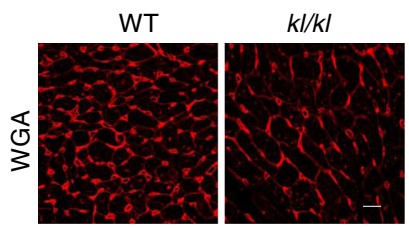

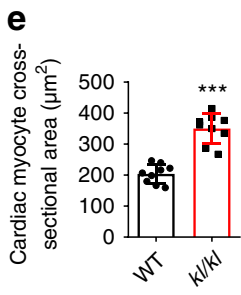

f

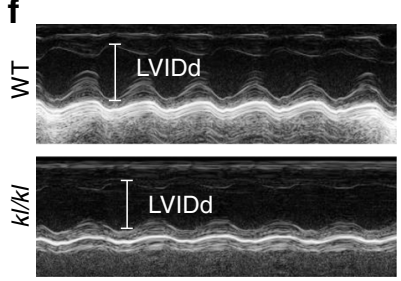

g

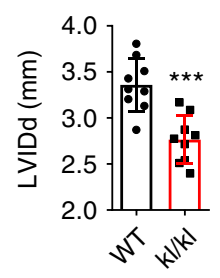

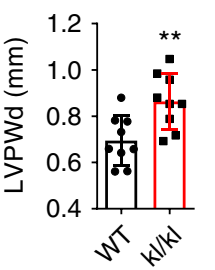

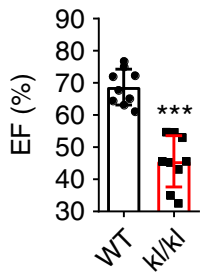

j
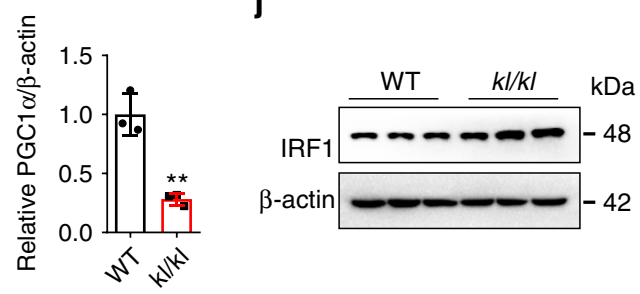

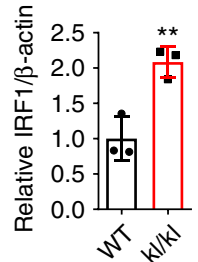

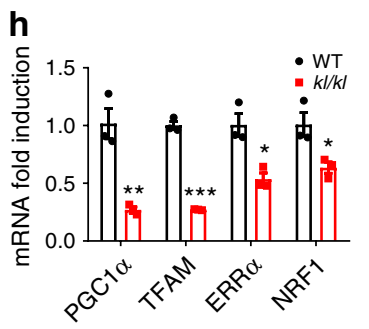

i
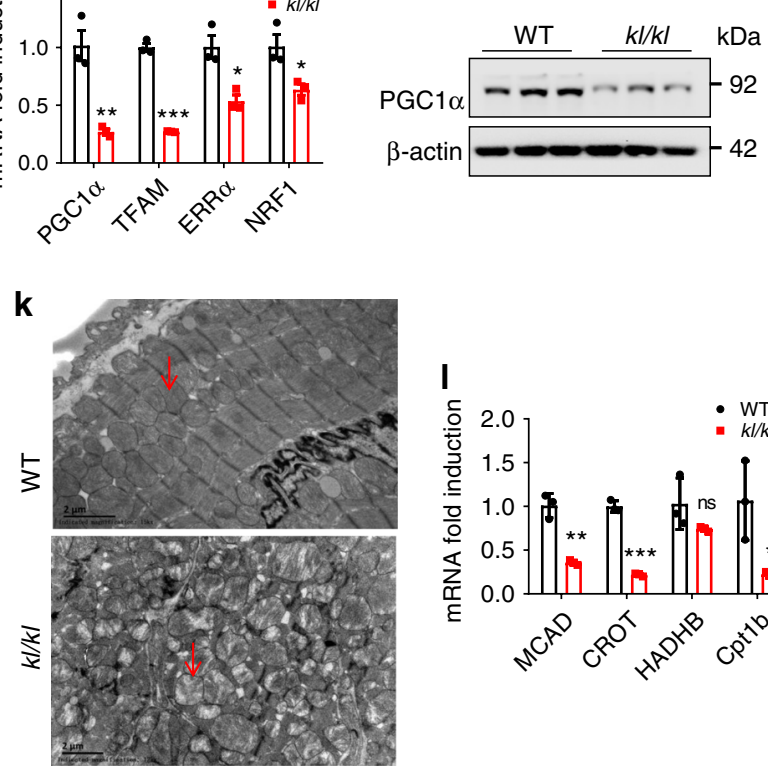

I
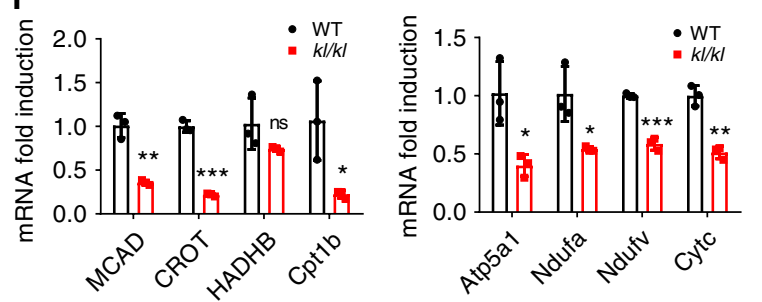

m

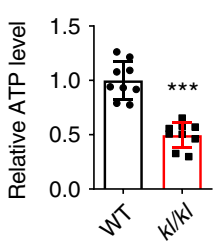

Fig. 7 Klotho deficiency leads to hyperphosphatemia, $\mathbf{L V H}$, and heart failure. a Serum phosphate levels of wild type (WT) and $k l / k l$ mice. $n=9$ mice per group. b The ratios of heart weight (HW) to body weight (BW), and HW to tibial length (TL) of mice in a. c, d Representative gross pathology (c HE staining, scale bar, $1 \mathrm{~mm}$ ) and WGA staining (d scale bar, $10 \mu \mathrm{m})$ of heart sections from WT and $\mathrm{kl} / \mathrm{kl}$ mice. e Cross-sectional surface area of individual cardiac myocytes from mice in d. f, $\mathbf{g}$ Echocardiography of LVIDd, LVPWd, and EF\% in WT and kl/kl mice. $\mathbf{h}-\mathbf{k}$ qPCR analysis of PGC1 $\alpha$ and its target genes (h), western blot analysis of PGC1 $\alpha(\mathbf{i})$, and IRF1 (j) expressions, representative images of TEM observation of mitochondria (k). Red arrows point to mitochondria. Scale bar, $2 \mu \mathrm{m}$, relative mRNA expression of metabolic genes (I), and relative ATP level $\mathbf{m}$ in the heart samples from WT and $k l / k l$ mice. $n=9(\mathbf{a}, \mathbf{b}, \mathbf{e}, \mathbf{g}, \mathbf{m})$, or $n=3(\mathbf{h}-\mathbf{I})$. Data are shown as mean \pm SD and were analyzed by a two-tailed unpaired $t$-test $(\mathbf{a}, \mathbf{b}, \mathbf{e}, \mathbf{g}-\mathbf{j}, \mathbf{I}-\mathbf{m}) .{ }^{\star} P<0.05,{ }^{\star \star} P<0.01$, $\star \star \star * P<0.001$.

effect existed between Pit1 and Pit2 in cardiomyocytes, consistent with a previous report ${ }^{43}$. Hence, it is necessary to knock down Pit1 and Pit2 synchronously to inhibit the effect of HP on the IRF1-PGC1 $\alpha$ axis and cardiomyocyte hypertrophy, which was confirmed in our study.

Although hyperphosphatemia, a typical serological feature of CKD patients, acts as an independent risk factor for cardiovascular death ${ }^{14,27}$, we are aware that altering phosphate homeostasis in vivo may lead to changes in FGF23, PTH, Klotho, and VitD, which can also contribute to myocardial hypertrophy and energy metabolism changes ${ }^{24,25}$. Therefore, it is difficult to exclude the roles of the above minerals and hormones in HPinduced effects in vivo. Previous studies have suggested that HP may also have an indirect effect on the cardiovascular system by elevating FGF2344, which can promote cardiac hypertrophy through activation of the FGFR-calcineurin-nuclear factor of the activated $T$ cells (NFAT) signaling pathway ${ }^{12}$. In this study, we found that HP-mediated alterations in the IRF1-PGC1 $\alpha$ axis were independent of the FGF23-FGFR signaling pathway. However, an additive effect between FGF23 and HP on inducing cardiac hypertrophy and energy metabolism changes was observed, indicating that end-stage renal disease (ESRD) or dialysis patients with hyperphosphatemia and high FGF23 levels may suffer more from myocardial hypertrophy. Because hyperphosphatemia can also increase the secretion of FGF23, it is important to control hyperphosphatemia in CKD patients to alleviate cardiac injury. 
a

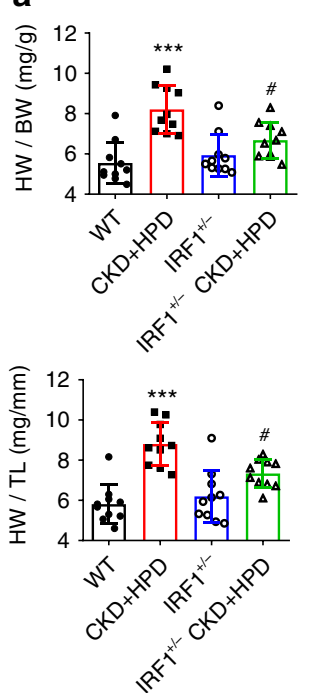

C

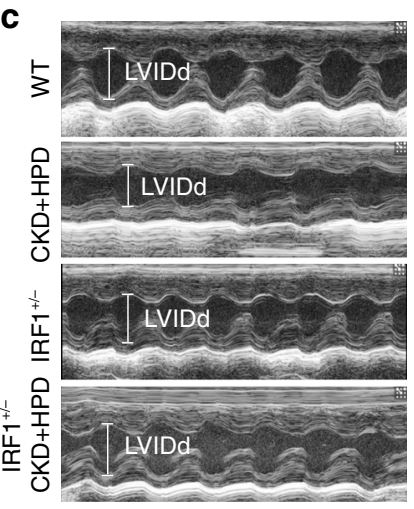

b WT

CKD+HPD
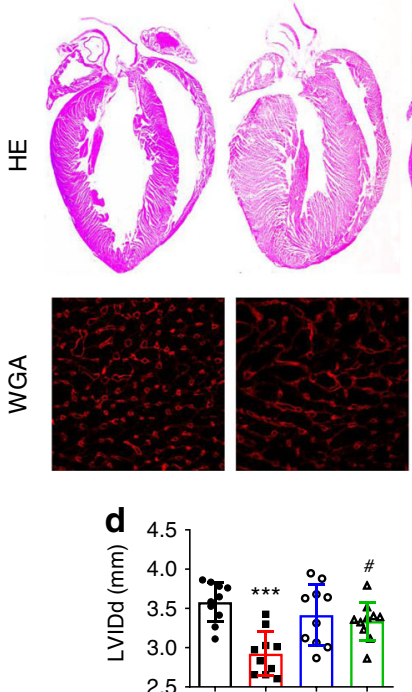

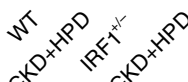

年

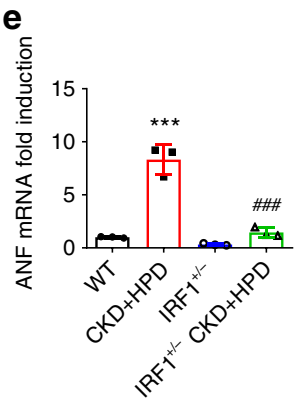

$\mathrm{IRF}^{+/-} \quad \mathrm{IRF}^{+/-} \mathrm{CKD}+\mathrm{HPD}$
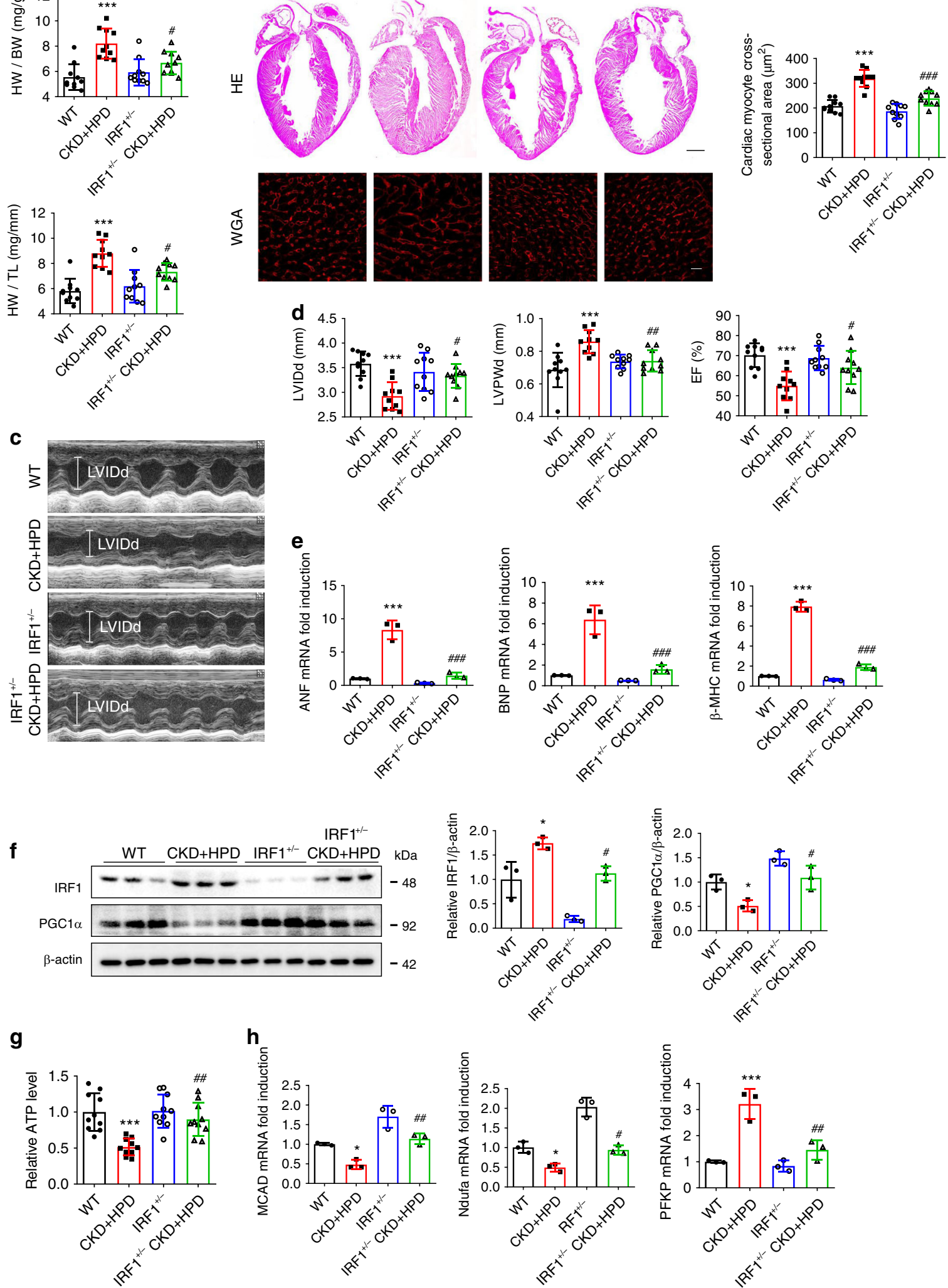

In addition, phosphate can interact with calcium, pyrophosphate, and fetuin-A, possibly contributing to CVD through precipitation ${ }^{45,46}$. Although we demonstrated that pyrophosphate or fetuin-A had no influence on the HP-mediated IRF1PGC1a axis (Supplementary Fig. 16), the harmful effect of HP on the cardiovascular system via interaction with calcium, pyrophosphate, and fetuin-A cannot be neglected. Despite the complex function of phosphate, the genetic knockdown of IRF1 was able to, at least partially, rescue HP-mediated cardiac energy metabolism remodeling and hypertrophy in vitro and in vivo, suggesting a distinctive and important role of the IRF1-PGC1a axis in CRS4. 
Fig. 8 Genetic knockdown of IRF1 protects against HPD-induced HF in CKD mice. a The ratios of HW to BW and HW to TL of WT, HPD-fed WT CKD, $\mathrm{IRF}^{+/}{ }^{-}$, and HPD-fed IRF1 ${ }^{+/}$- CKD mice for 12 weeks. $n=10$ mice per group. b Representative gross pathology of heart sections (HE staining, upper panel, scale bar, $1 \mathrm{~mm}$ ), WGA staining of the left ventricle of heart sections (lower panel, scale bar, $10 \mu \mathrm{m}$ ), and cross-sectional surface area of individual cardiac myocytes from mice in a. c, d Echocardiographic detection of LVIDd, LVPWd, and EF\% in mice in a. e-h qPCR analysis of hypertrophic genes expression (e), western blot analysis of IRF1 and PGC1 $\alpha$ expressions (f), relative ATP level (g), and relative mRNA expression of metabolic genes (h) of heart lysates from the mice in $\mathbf{a}$. $\beta$-actin was taken as the loading control. $n=10$ (a-d, $\mathbf{g})$, or $n=3(\mathbf{e}, \mathbf{f}, \mathbf{h})$. Data are shown as mean \pm SD and were analyzed by one-way ANOVA. ${ }^{\star} P<0.05,{ }^{\star \star} P<0.01,{ }^{\star \star \star} P<0.001$ versus WT. ${ }^{*} P<0.05,{ }^{\# \# P}<0.01, \# \# \# P<0.001$ versus CKD $+\mathrm{HPD}$.

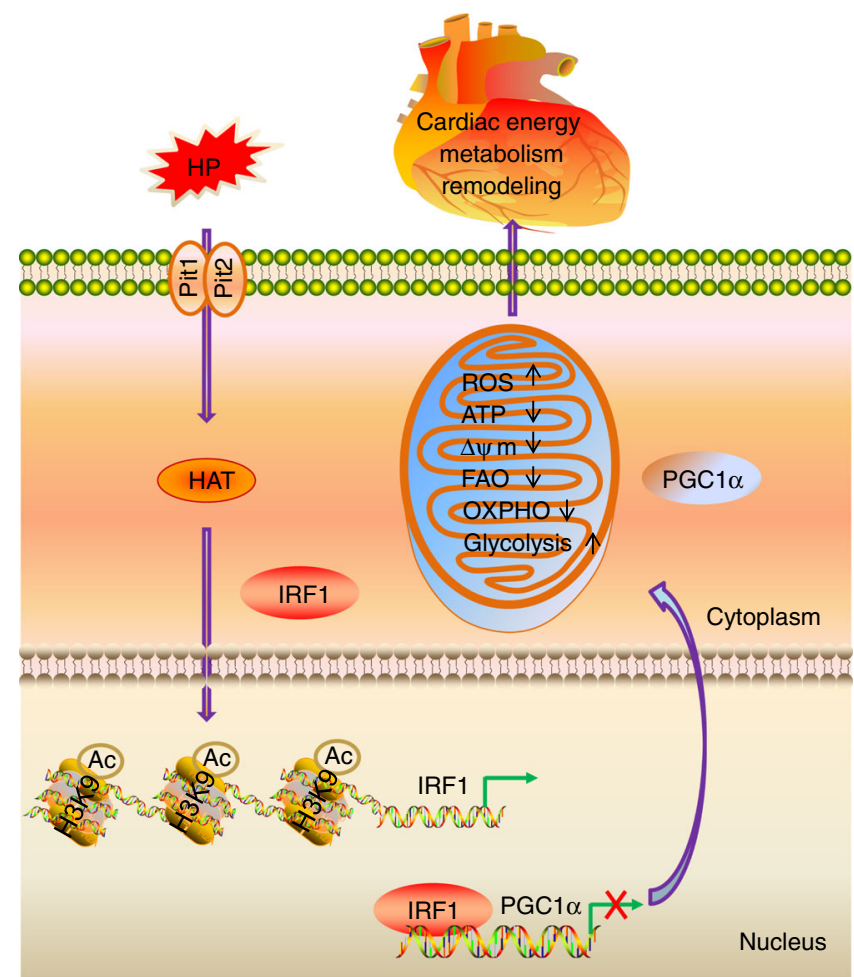

Fig. 9 A proposed model of the mechanism underlying myocardial energy metabolism remodeling and $\mathbf{H F}$ induced by HP. High phosphate (HP) enters cardiomyocytes via Pit1 and Pit2, and induces IRF1 expression through acetylating histone H3K9 in the IRF1 promoter region, during which histone acetyltransferases (HAT) are involved. HP-induced IRF1 inhibits PGC1 $\alpha$ expression via directly binding to its promoter region ( -632 to -612). PGC1 $\alpha$ downregulation contributes to HP-induced mitochondrial dysfunction and myocardial energy metabolism remodeling. Restoration of PGC1 $\alpha$ expression or knockdown of IRF1 protects against CKD-associated myocardial energy metabolism remodeling and HF.

In summary, our findings demonstrated that HP-induced alterations in the IRF1-PGC1a signaling pathway had an important role in the pathogenesis of CRS4 and restoration of PGC1a expression or knockdown of IRF1 improved energy metabolic dysfunction and HF under uremic milieu. Our study suggests that control of hyperphosphatemia or targeted intervention on HP-mediated IRF1 elevation and PGC1 $\alpha$ downregulation could be a potential therapy for reducing the risk of cardiovascular death in CKD patients.

\section{Methods}

Patient samples collection. A total of 213 predialysis CKD patients (aged 18-80 years old) were enrolled in this study (Department of Nephrology of Xinqiao Hospital, Chongqing, China). The pre-established exclusion criteria were diabetes, pregnancy, HIV, polycystic kidney disease, renal cancer, and recent immunosuppressive therapy. The basic information and laboratory parameters including serum inorganic phosphate and eGFR of the enrolled patients were collected. Echocardiograms were performed on the first day of hospitalization using twodimensional M-mode. EF\%, ventricular dimension and wall thickness were detected at end-diastole and end-systole using IE33-5S (Philips Medical System). LVMI was calculated by indexing left ventricular mass to height ${ }^{2}$. Serum samples of three healthy donors and three CKD patients at stage 5 were collected for later experiments. Informed written consent was provided by all participants. All human studies, including collection and use of samples, were approved by the Ethics Committee of Xinqiao Hospital of the Army Medical University (No. 2018-00601 ), and the study design and conduct complied with all relevant regulations regarding the use of human study participants and was conducted in accordance to the criteria set by the Declaration of Helsinki, and the study is compliant with the guidance of the Ministry of Science and Technology (MOST) for the Review and Approval of Human Genetic Resources.

Animal treatment. IRF1 knockout mice were obtained from Jackson Laboratory (Bar Harbor, ME, USA), which were backcrossed to C57BL/6J background to produce heterozygous IRF1 ${ }^{+/-}$mice. $k l / k l$ mice were provided by Jun Gu (State Key Laboratory of Protein and Plant Gene Research, College of Life Science, Peking University, Beijing, China), and the mice were backcrossed to C57BL/6J background for more than 6 generations. C57BL/6J and Balb/c mice were purchased from Beijing Huafukang Bioscience (Beijing, China). Male Balb/c, WT C57BL/6J and IRF $1^{+1-}$ mice of 8 -week-old were inflicted with $2 / 3$ electrocoagulation of the right renal cortex (the lower and upper thirds of the kidney) and received left total nephrectomy two weeks later to construct $5 / 6$ nephrectomy CKD model, which is in accordance with the clinical manifestations of human ESRD patients and described in multiple reports including our own studies ${ }^{11,47}$. Mice underwent laparotomy without kidney operation served as a sham group. Then the mice were randomly separated into the following groups and were blinded to the investigators who helped collect samples. (1) Sham and CKD Balb/c mice fed with normal diet for 8 weeks. (2) Sham, CKD, CKD + HPD (fed with $2 \%$ HPD) Balb/c mice for 12 weeks. (3) Sham, CKD + HPD, Nam (intraperitoneally injected with $400 \mathrm{mg} / \mathrm{kg} /$ day. Sigma, St. Louis, MO, USA), CKD + HPD + Nam Balb/c mice for 12 weeks. (4) WT C57BL/6J, WT CKD + HPD, IRF1 $1^{+l-}, \mathrm{IRF}^{+/-} \mathrm{CKD}+\mathrm{HPD}$ mice for 12 weeks. Male C57BL/6J mice of 8-week-old without surgery were divided into the following groups. (1) WT and $k l / k l$ mice. (2) WT and IRF1 ${ }^{-1-}$ mice. (3) WT and IRF1 ${ }^{-1-}$ mice fed with or without HPD for 12 weeks. (4) Control and adenineinduced CKD mice (C57BL/6J mice) fed with a normal diet containing $0.25 \%$ adenine (Sigma) or not for 8 weeks. All animals were housed four to five per cage under a $12: 12 \mathrm{~h}$ light:dark cycle at $25^{\circ} \mathrm{C}$, with a humidity of $40-70 \%$.

High-resolution echocardiography was performed under anesthesia, and left ventricular internal diameter at diastole (LVIDd), left ventricular posterior wall thickness at diastole (LVPWd), EF\% and short-axis M-mode views were recorded by the Vevo 770 Echocardiography Imaging System (VisualSonics, Toronto, ON, Canada). Mice were euthanized by carbon dioxide inhalation at appointed times, and the heart weight (HW), body weight (BW), and tibial length (TL) were recorded. Serum phosphate, BUN, and Scr were measured. The hearts were harvested for three parts: (1) perfused with $4 \%$ paraformaldehyde for hematoxylineosin (HE) and Wheat Germ Agglutinin (WGA) staining; (2) fixed in 2.5\% glutaraldehyde at $4{ }^{\circ} \mathrm{C}$ for TEM observation; (3) froze in liquid nitrogen for qPCR and western blot analysis. All animal procedures were approved by the Animal Care and Use Committee of the Army Medical University, and were performed in accordance with the guidelines established by the Committee.

Enzyme-linked immunosorbent assay. Enzyme-linked immunosorbent assay (ELISA) was performed using ELISA kits according to the manufacturer's instructions. Mouse ELISA kits detecting serum intact FGF23, Cterminal FGF23 and PTH were bought from Immutopics (San Clemente, CA, USA). Mouse VitD ELISA kit was obtained from AbebioSience (Wuhan, China). Mouse klotho ELISA kit was acquired from Cusabio (Cologne, Germany).

Cell culture. Rat embryonic cardiomyoblast cell line H9c2 was obtained from American Type Culture Collection (Manassas, VA, USA). Primary neonatal rat ventricular myocytes (NRVMs) were isolated as we and others previously described $^{11,48}$. Briefly, the left ventricles of 1-day-old Sprague-Dawley rats (Beijing Huafukang Bioscience) were collected and digested with trypsin (Thermo Scientific, Waltham, MA, USA) at room temperature, and then incubated with $0.1 \%$ collagenase II (Solarbio Life Sciences, Beijing, China) for $30 \mathrm{~min}$ at $37^{\circ} \mathrm{C}$. Cells were filtered through a cell strainer ( $70 \mu \mathrm{m}$, BD Falcon, Lincoln Park, NJ, USA), and centrifuged at $1500 \mathrm{rpm}$ for $10 \mathrm{~min}$. Then, the cells were resuspended in Dulbecco's Modified Eagle's Medium (DMEM, Gibco, Grand Island, NY, USA) 
supplemented with $15 \%$ fetal bovine serum (FBS, Gibco) for $1 \mathrm{~h}$, and in DMEM supplemented with 10 FBS and $0.1 \mathrm{mM}$ bromodeoxyuridine (BrdU) for $48 \mathrm{~h}$. Finally, both NRCMs and H9c2 cells were cultured in DMEM supplemented with $10 \%$ FBS and $1 \%(\mathrm{v} / \mathrm{v})$ penicillin/streptomycin (Beyotime, Shanghai, China) at $37^{\circ} \mathrm{C}$ and $5 \% \mathrm{CO}_{2}$.

Inorganic phosphate measurement. Intracellular inorganic phosphate in the supernatant of cell lysate was measured using the molybdate blue method with an inorganic phosphate assay kit (Jiancheng Bioengineering Institute, Nanjing, China) according to the manufacturer's instructions. The corresponding protein concentration was determined using the BCA kit (Beyotime). The phosphate level of each sample was normalized against the protein content, and then compared with the control group for a relative intracellular phosphate level.

Reverse transcription and qPCR. All tissue samples were collected on ice and flash-frozen using liquid nitrogen immediately. Samples were kept in liquid nitrogen for long-term storage, and were reserved in $-80^{\circ} \mathrm{C}$ refrigerator for shortterm use. After isolation using Trizol (Invitrogen, Carlsbad, CA, USA) method, RNA was kept in $0.1 \%$ diethyl pyrocarbonate (DEPC) water, and the $\mathrm{OD}_{260} / \mathrm{OD}_{280}$ of RNA was detected using a NanoDrop 2000 spectrophotometer (Thermo Scientific). Only when the $\mathrm{OD}_{260} / \mathrm{OD}_{280}$ of RNA was between 1.8 and 2.0 did the RNA was used for subsequent reverse transcription using a reverse transcription kit (Promega, Madison, WI, USA). Real-time PCR was performed with SYBR Green qPCR kit (Takara, Dalian, China). The primers for qPCR are listed in Supplementary Tables 3 and 4 .

Western blot. Total protein was extracted with cell lysis buffer (Beyotime) supplemented with protease and phosphatase inhibitor cocktail (Roche Diagnostics $\mathrm{GmbH}$, Mannheim, Germany), and the concentration was measured with BCA kit (Beyotime). The proteins were separated with 12\% SDS-PAGE and transferred to PVDF membranes (ThermoScientific). The membranes were separately incubated with primary antibodies overnight at $4{ }^{\circ} \mathrm{C}$, and anti-rabbit (A7016) or anti-mouse (A0216) HRP-conjugated secondary antibodies (Beyotime) for $1 \mathrm{~h}$ at room temperature ${ }^{49}$. Subsequently, the membranes were washed and the signals were visualized with Clarity ECL Substrate (Bio-Rad, Hercules, CA, USA). Gray-scale results were normalized against $\beta$-actin for semiquantitative analysis. The antibodies against IRF1 (sc-514544x), PPAR $\alpha$ (sc-130640), and $\beta$-actin (sc-47778) were bought from Santa Cruz Biotechnology (Dallas, TX, USA). The antibodies against PGC1a (ab54481), Pit1 (ab10545), Pit2 (ab191182), phosphorylated FGFR1 (pFGFR1, ab59194), total FGFR1 (t-FGFR1, ab824), phosphorylated FGFR4 (pFGFR4, ab192589), total FGFR4 (t-FGFR4, ab41948), NRF1 (ab175932), and TFAM (ab131607) were obtained from Abcam Biotechnology (Cambridge, MA, USA). All of the antibodies were diluted 1:1000 for western blot. Uncropped and unprocessed scans of all blots are provided in Supplementary Figs. 17-21 in the Supplementary Information.

ATP measurement. After cell lysis and centrifugation, the supernatant was determined using an ATP Determination Kit from Invitrogen company (A22066) according to the manufacturer's instructions. The cellular ATP level was calculated according to the standard curve using an ATP reference standard. The corresponding protein concentration was tested using a BCA kit (Beyotime). ATP level $(\mathrm{nmol})$ was normalized to total protein content $(\mathrm{mg})$. Finally, each sample was compared with the control group for a relative ATP level.

ROS detection. After HP treatment, $\mathrm{H} 9 \mathrm{c} 2$ cells were washed with serum-free DMEM and incubated with CM-H2DCF-DA at $37^{\circ} \mathrm{C}$ for $20 \mathrm{~min}$, and analyzed using Accuri C6 flow cytometry (BD Biosciences, San Jose, CA, USA). A figure exemplifying the gating strategy is provided in Supplementary Fig. 22.

Mitochondrial DNA analysis. Total DNA, including chromosomal (B2M) and mitochondrial (D-loop) DNA, was extracted from H9c2 cells using a DNA extraction kit (Takara) following the manufacturer's instructions. Real-time PCR was carried out using an SYBR Green qPCR kit (Takara). Chromosomal DNA served as an internal control. The primers are shown in Supplementary Table 5.

Mitochondrial membrane potential. $\mathrm{H} 9 \mathrm{c} 2$ cells were treated with control or HP for $24 \mathrm{~h}$. The cells were incubated with JC- 1 at $37^{\circ} \mathrm{C}$ for $20 \mathrm{~min}$, washed twice with JC-1 buffer, and placed in the culture medium on ice. Cells were imaged using a confocal fluorescence microscope (Leica, Mannheim, Germany) or harvested for flow cytometry analysis (BD Biosciences).

Transmission electron microscopy. Heart tissues of mice were harvested and fixed in $2.5 \%$ glutaraldehyde overnight at $4{ }^{\circ} \mathrm{C}$ and post fixed with $2 \%$ osmium tetroxide for $1 \mathrm{~h}$ at $37^{\circ} \mathrm{C}$. The samples were gradiently dehydrated in acetone $(50 \%$, $70 \%, 90 \%$, and $100 \%$ separately) and embedded in epoxy resins. The ultrathin section was stained with uranyl acetate/lead citrate for visualization using a TEM (JEM-1400PLUS, Japan) ${ }^{49}$.
Mitochondrial oxygen consumption rate analysis. OCR was assessed using the Seahorse XF96 Extracellular Flux Analyzer (Agilent Technologies). Cells were seeded quadruplicately in cell culture microplates (Agilent Technologies, Santa Clara, CA, USA) and treated with control or HP for $24 \mathrm{~h}$. One hour prior to the assay, the culture medium was replaced by base medium (Agilent Technologies) supplemented with $1 \mathrm{mM}$ sodium pyruvate, $2 \mathrm{mM}$ glutamine, and $10 \mathrm{mM}$ glucose (Agilent Technologies), and then cells were incubated at $37^{\circ} \mathrm{C}$ without $\mathrm{CO}_{2}$. After three basal respiration measurements without additives, the ATP synthase inhibitor $(1 \mu \mathrm{M}$ oligomycin) was added, followed by subsequent mitochondrial uncoupler $(1 \mu \mathrm{M}$ FCCP $)$, and complex I and III inhibitors $(1 \mu \mathrm{M}$ rotenone and $1 \mu \mathrm{M}$ antimycin A), respectively. Three separate measurements were recorded after the reagents (all bought from Agilent Technologies) were injected.

Laser scanning confocal microscopy. After treatment, cardiac cells were fixed with $4 \%$ paraformaldehyde for $20 \mathrm{~min}$ and stained with $\alpha$-actinin (sc-17829, Santa Cruz Biotechnology, diluted 1:50) at $4{ }^{\circ} \mathrm{C}$ overnight. After washed with PBS, the cells were incubated with FITC-conjugated goat anti-mouse antibody (A0568, Beyotime, diluted 1:300) in the dark. Heart tissues were stained with WGA Alexa Fluor $^{\text {rt }} 555$ Conjugate $(500 \mu \mathrm{g} / \mathrm{ml}, \mathrm{W} 32464$, Thermo Scientific) at room temperature for $10 \mathrm{~min}$ and washed with PBS four times. Cells and tissue sections were imaged using a confocal fluorescence microscope (Leica).

Microarray analysis. Total RNA was extracted from the heart samples of sham and CKD mice for microarray analysis using Affymetrix Clariom S array (Affymetrix, Santa Clara, CA, USA), robust multiaverage, GO and KEGG pathway enrichment. Fold change $>1.5$ and $P<0.05$ was defined as differentially expressed.

Construction of overexpression plasmids. Full-length cDNA sequence of rat IRF1, PGCla, NRF1, and TFAM was obtained from Pubmed. The corresponding primers, restriction enzymes and vectors are listed in Supplementary Table 6. PCR was performed to obtain the target gene fragments. After digestion, recombination, transformation, culture of the bacteria with recombinant plasmids, and further culture of the positive monoclones, the bacteria were identified by Beijing Genomics Institute (Beijing, China).

Overexpression or silence of target genes. $\mathrm{H} 9 \mathrm{c} 2$ cells were separately transfected with siRNAs (IRF1, Pit1, and Pit2), or overexpression plasmids (IRF1, PGCla, NRF1, and TFAM) using Lipofectamine 2000 (Invitrogen) in OptiMEM (Hyclone, Logan, Utah, USA), according to the manufacturer's protocol. Then, cells were treated with HP for another $24 \mathrm{~h}$, and were harvested for the subsequent experiments. Scramble siRNA and siRNAs targeted to IRF1, Pit1, and Pit2 (listed in Supplementary Table 7) were synthesized by Sangon (Shanghai, China).

Construction of reporter plasmids and point mutation. Putative IRF1 binding sites in the PGCla promoter region are listed in Supplementary Table 1. Various lengths of the rat PGCl $\alpha$ promoter region were amplified by PCR using the genomic DNA of $\mathrm{H} 9 \mathrm{c} 2$ cells as a template. The corresponding primers are listed in Supplementary Table 8 . The fragments including PGC1 $\alpha-1703(-1703$ to +188$)$, PGC1a-1524 (-1524 to +188), PGC1 $\alpha-985(-985$ to +188$)$, PGC1 $\alpha-719$ ( -719 to $+188)$, PGC1 $\alpha-348$ ( -348 to +188$)$, and PGC1 $\alpha-183(-183$ to +188$)$ were separately cloned into a pGL3-basic vector (Promega) after digestion with HindIII, and the recombinant reporter plasmids were separately named as pGL3-PGC1 $\alpha$ P6, pGL3-PGC1 $\alpha$-P5, pGL3-PGC1 $\alpha$-P4, pGL3-PGC1 $\alpha-P 3$, pGL3-PGC1 $\alpha-P 2$, and pGL3-PGC1 $\alpha-P 1$. pGL3-PGC1 $\alpha-M 3$, containing point mutations in the IRF1 binding element (AATAGGGAGGAGAGGGAAGGA, the mutated bases are underlined), was generated with Mutan $\overline{B E S T}$ kit (Takara) using pGL3-PGC1a-P3 $(-719$ to +188$)$ as a template. Negative control mutation plasmid pGL3-PGC1aM4, containing point mutations in the IRF1 binding element (ATATAA-

GAAGGGAGGGGGGGG, the mutated bases are underlined), was also generated, using $\mathrm{pG}$ L3-PGC1 $\alpha-\mathrm{P} 4(-985$ to +188$)$ as a template.

Dual-luciferase reporter assay. The recombinant reporter plasmids were cotransfected with pRL-TK vector (Promega) into H9c2 cells using Lipofectamine 2000 (Invitrogen) in OptiMEM (Hyclone). Then, the cells were treated with control or HP for another $24 \mathrm{~h}$. Luciferase activity was detected using the Dual-luciferase reporter assay system (Promega). The firefly luciferase activity was normalized against Renilla activity. All transfection experiments were performed three times in triplicate.

Chromatin immunoprecipitation. H9c2 cells were treated with control or HP for $24 \mathrm{~h}$, and then fixed with $1 \%$ formaldehyde for $10 \mathrm{~min}$. The cells were lyzed in SDS lysis buffer. The chromatin was sonicated to shear DNA to an average length between 200 to $1000 \mathrm{bp}$, and immunoprecipitated with $2 \mu \mathrm{g}$ antibody against IRF1 (sc-514544x, Santa Cruz Biotechnology), taking IgG as a negative control. The precipitated DNA was amplified by PCR and qPCR with the primers $(-709$ to -511$)$ that cover the IRF1 binding sites $(-632$ to -612$)$. Primers $(-1711$ to -1598$)$ without IRF1 binding sites served as a negative control, while the total DNA (Input) served as a positive control. For acetylation detection, the sonicated DNA was immunoprecipitated with $2 \mu \mathrm{g}$ antibody against H3 (Lys9) 
(\#9671) or H4 (Lys12) (\#13944) (Cell Signaling Technology, Danvers, MA, USA), taking IgG as a negative control. The primers for ChIP are listed in Supplementary Table 9.

Methylation-specific PCR. Genomic DNA of H9c2 cells was modified with bisulfate using EZ DNA Methylation Gold Kit (Zymo Research, Irvine, CA) according to the manufacturer's instructions. The primers that recognize the methylated and unmethylated CpG island sites in the IRF1 promoter region were designed by MethPrimer software ${ }^{50}$ (http://www.urogene.org/methprimer/) and listed in Supplementary Table 10. The PCR products of genomic DNA without bisulfate modification served as a positive control (Input).

Statistical analysis. All continuous characteristics were plotted by bar charts (means and standard deviations). Comparisons of indicators that follow normal distribution between two groups were analyzed by two independent sample $t$-test, and that among multiple groups were tested by one-way analysis of variance (ANOVA). Considering treatment time factors, comparisons of indicators between treatment groups were examined using analysis of variance of factorial design. In the human study, continuous characteristics that obey non-normal distribution were drawn by box plots (medians and interquartiles). Spearman rank correlation was used to analyze the correlation between phosphate levels and eGFR, and partial correlation analyses were performed to examine the associations of EF and LVMI with phosphate levels after controlling eGFR. Kruskal-Wallis $H$ test was used to compare the indicators among three tertiles of the serum phosphate levels. Statistical analyses were performed using Graphpad Prism 8.0 and SPSS 21.0, and all tests were two-tailed with a significant level set at $P<0.05$.

Reporting summary. Further information on research design is available in the Nature Research Reporting Summary linked to this article.

\section{Data availability}

All raw data of microarray analyses have been submitted to Gene Expression Omnibus (GEO) database (accession no. GSE143031). Source data are provided with this paper Other data that support the findings of this study are available within the article and Supplementary files. Source data are provided with this paper.

Received: 14 March 2019; Accepted: 18 August 2020;

Published online: 16 September 2020

\section{References}

1. Webster, A. C., Nagler, E. V., Morton, R. L. \& Masson, P. Chronic kidney disease. Lancet 389, 1238-1252 (2017).

2. Tuegel, C. \& Bansal, N. Heart failure in patients with kidney disease. Heart 103, 1848-1853 (2017)

3. Chuppa, S. et al. MicroRNA-21 regulates peroxisome proliferator-activated receptor alpha, a molecular mechanism of cardiac pathology in cardiorenal syndrome type 4. Kidney Int. 93, 375-389 (2018).

4. Schefold, J. C., Filippatos, G., Hasenfuss, G., Anker, S. D. \& von Haehling, S. Heart failure and kidney dysfunction: epidemiology, mechanisms and management. Nat. Rev. Nephrol. 12, 610-623 (2016).

5. Bansal, N. et al. Absolute rates of heart failure, coronary heart disease, and stroke in chronic kidney disease: an analysis of 3 community-based cohort studies. JAMA Cardiol. 2, 314-318 (2017).

6. Noordali, H., Loudon, B. L., Frenneaux, M. P. \& Madhani, M. Cardiac metabolism-a promising therapeutic target for heart failure. Pharmacol. Ther. 182, 95-114 (2018)

7. Doenst, T., Nguyen, T. D. \& Abel, E. D. Cardiac metabolism in heart failure: implications beyond ATP production. Circul. Res. 113, 709-724 (2013).

8. Neubauer, S. The failing heart-an engine out of fuel. N. Engl. J. Med. 356, 1140-1151 (2007).

9. Higuchi, T. et al. Levocarnitine improves cardiac function in hemodialysis patients with left ventricular hypertrophy: a randomized controlled trial. Am. J. Kidney Dis. 67, 260-270 (2016).

10. Go, A. S., Chertow, G. M., Fan, D., McCulloch, C. E. \& Hsu, C.-Y. Chronic kidney disease and the risks of death, cardiovascular events, and hospitalization. N. Engl. J. Med. 351, 1296-1305 (2004).

11. Yang, K. et al. Klotho protects against indoxyl sulphate-induced myocardial hypertrophy. J. Am. Soc. Nephrol. 26, 2434-2446 (2015).

12. Faul, C. et al. FGF23 induces left ventricular hypertrophy. J. Clin. Investig. 121, 4393-4408 (2011).

13. Bennett, B. J. et al. Trimethylamine-N-oxide, a metabolite associated with atherosclerosis, exhibits complex genetic and dietary regulation. Cell Metab. 17, 49-60 (2013).
14. Stevens, K. K., Patel, R. K., Mark, P. B., Delles, C. \& Jardine, A. G. Phosphate as a cardiovascular risk factor: effects on vascular and endothelial function. Lancet 385, S10 (2015).

15. You, L. et al. High levels of serum beta2-microglobulin predict severity of coronary artery disease. BMC Cardiovasc. Disord. 17, 71 (2017).

16. Lekawanvijit, S., Kompa, A. R., Wang, B. H., Kelly, D. J. \& Krum, H. Cardiorenal syndrome: the emerging role of protein-bound uremic toxins. Circ. Res. 111, 1470-1483 (2012).

17. Tran, M. T. et al. PGC1alpha drives NAD biosynthesis linking oxidative metabolism to renal protection. Nature 531, 528-532 (2016).

18. Schilling, J. \& Kelly, D. P. The PGC-1 cascade as a therapeutic target for heart failure. J. Mol. Cell. Cardiol. 51, 578-583 (2011).

19. Dou, L. et al. The uremic solutes p-cresol and indoxyl sulfate inhibit endothelial proliferation and wound repair. Kidney Int. 65, 442-451 (2004).

20. GC, Rowe, Jiang, A. \& Arany, Z. PGC-1 coactivators in cardiac development and disease. Circul. Res. 107, 825-838 (2010).

21. Hathorn, T., Snyder-Keller, A. \& Messer, A. Nicotinamide improves motor deficits and upregulates PGC-1alpha and BDNF gene expression in a mouse model of Huntington's disease. Neurobiol. Dis. 41, 43-50 (2011).

22. Jiang, D.-S. et al. Interferon regulatory factor 1 is required for cardiac remodeling in response to pressure overload. Hypertension 64, 77-86 (2014).

23. Tan, X., Xu, X., Zeisberg, M. \& Zeisberg, E. M. DNMT1 and HDAC2 cooperate to facilitate aberrant promoter methylation in inorganic phosphateinduced endothelial-mesenchymal transition. PLoS ONE 11, e0147816 (2016).

24. Razzaque, M. S. The role of Klotho in energy metabolism. Nat. Rev. Endocrinol. 8, 579-587 (2012).

25. $\mathrm{Hu}, \mathrm{M}$. C. et al. Klotho and phosphate are modulators of pathologic uremic cardiac remodeling. J. Am. Soc. Nephrology 26, 1290-1302 (2015).

26. Chronic Kidney Disease Prognosis Consortium et al. Association of estimated glomerular filtration rate and albuminuria with all-cause and cardiovascular mortality in general population cohorts: a collaborative meta-analysis. Lancet 375, 2073-2081 (2010).

27. Chang, A. R. \& Anderson, C. Dietary Phosphorus Intake and the Kidney. Annu Rev. Nutr. 37, 321-346 (2017).

28. Yamamoto, K. T. et al. Dietary phosphorus is associated with greater left ventricular mass. Kidney Int. 83, 707-714 (2013).

29. Maizel, J. et al. Effects of sevelamer treatment on cardiovascular abnormalities in mice with chronic renal failure. Kidney Int. 84, 491-500 (2013).

30. Tonelli, M., Pannu, N. \& Manns, B. Oral phosphate binders in patients with kidney failure. N. Engl. J. Med. 362, 1312-1324 (2010).

31. Abdurrachim, D. et al. Good and bad consequences of altered fatty acid metabolism in heart failure: evidence from mouse models. Cardiovasc. Res. 106, 194-205 (2015).

32. Lopaschuk, G. D., Ussher, J. R., Folmes, C. D., Jaswal, J. S. \& Stanley, W. C. Myocardial fatty acid metabolism in health and disease. Physiol. Rev. 90 207-258 (2010).

33. Hirotsu, Y., Hataya, N., Katsuoka, F. \& Yamamoto, M. NF-E2-related factor 1 (Nrf1) serves as a novel regulator of hepatic lipid metabolism through regulation of the Lipin1 and PGC-1beta genes. Mol. Cell Biol. 32, 2760-2770 (2012).

34. Hirotsu, Y. et al. Transcription factor NF-E2-related factor 1 impairs glucose metabolism in mice. Genes Cells 19, 650-665 (2014).

35. Eckert, M., Meek, S. E. M. \& Ball, K. L. A novel repressor domain is required for maximal growth inhibition by the IRF-1 tumor suppressor. J. Biol. Chem. 281, 23092-23102 (2006).

36. Fragale, A. et al. IFN regulatory factor-1 negatively regulates CD4+ CD25+ regulatory $\mathrm{T}$ cell differentiation by repressing Foxp3 expression. J. Immunol. 181, 1673-1682 (2008)

37. Yu, Q. et al. IL-18 induction of osteopontin mediates cardiac fibrosis and diastolic dysfunction in mice. Am. J. Physiol. Heart Circ. Physiol. 297, H76-H85 (2009).

38. Jiang, D. S. et al. Interferon regulatory factor 7 functions as a novel negative regulator of pathological cardiac hypertrophy. Hypertension 63, 713-722 (2014).

39. Tan, X., Xu, X., Zeisberg, E. M. \& Zeisberg, M. High inorganic phosphate causes DNMT1 phosphorylation and subsequent fibrotic fibroblast activation. Biochem. Biophys. Res. Commun. 472, 459-464 (2016)

40. Li, S. J. et al. Activated p300 acetyltransferase activity modulates aortic valvular calcification with osteogenic transdifferentiation and downregulation of Klotho. Int. J. Cardiol. 232, 271-279 (2017).

41. Xiao, D. et al. Inhibition of DNA methylation reverses norepinephrineinduced cardiac hypertrophy in rats. Cardiovasc. Res. 101, 373-382 (2014).

42. Gusterson, R. J., Jazrawi, E., Adcock, I. M. \& Latchman, D. S. The transcriptional co-activators CREB-binding protein (CBP) and p300 play a critical role in cardiac hypertrophy that is dependent on their histone acetyltransferase activity. J. Biol. Chem. 278, 6838-6847 (2003).

43. Crouthamel, M. H. et al. Sodium-dependent phosphate cotransporters and phosphate-induced calcification of vascular smooth muscle cells: redundant 
roles for PiT-1 and PiT-2. Arterioscler. Thromb. Vasc. Biol. 33, 2625-2632 (2013).

44. Leifheit-Nestler, M. et al. Impact of altered mineral metabolism on pathological cardiac remodeling in elevated fibroblast growth factor 23. Front. Endocrinol. 9, 333 (2018).

45. Lomashvili, K. A., Narisawa, S., Millan, J. L. \& O’Neill, W. C. Vascular calcification is dependent on plasma levels of pyrophosphate. Kidney Int. 85, 1351-1356 (2014).

46. Ketteler, M. et al. Association of low fetuin-A (AHSG) concentrations in serum with cardiovascular mortality in patients on dialysis: a cross-sectional study. Lancet 361, 827-833 (2003).

47. Barata, K., Yoshida, M., Hokao, R. \& Maekawa, A. Sequential alterations in clinical biochemical indicators of renal function in 5/6 nephrectomized rats-basic study for renal toxicity using $5 / 6$ nephrectomized rats. J. Toxicol. Sci. 23, 433-442 (1998).

48. Louch, W. E., Sheehan, K. A. \& Wolska, B. M. Methods in cardiomyocyte isolation, culture, and gene transfer. J. Mol. Cell Cardiol. 51, 288-298 (2011).

49. Huang, Y. et al. Identification of a fluorescent small-molecule enhancer for therapeutic autophagy in colorectal cancer by targeting mitochondrial protein translocase TIM44. Gut 67, 307-319 (2018).

50. Li, L. C. \& Dahiya, R. MethPrimer: designing primers for methylation PCRs. Bioinformatics 18, 1427-1431 (2002).

\section{Acknowledgements}

This study was supported by research grants from National Key R\&D Program of China (2018YFC1312700, 2017YFA0106600), the Natural Science Foundation of China (Nos. 81873605, 81800468, 81700379, 81400747), Personal Training Program for Clinical Medicine Research of Army Medical University (2018XLC1007), and Frontier Specific Project of Xinqiao Hospital (2018YQYLY004).

\section{Author contributions}

Y.H. performed the experiments, analyzed the data, and drafted the manuscript; S.W., J.Z., Y.L., C.D., K.Y., X.B., and M.L. took part in the molecular study; W.H., K.W., J.X., S.W., and Y.W. contributed to human samples collection and data analysis; L.N., C.L.,
D.Z., J.G., and C.Z. participated in the animal study and paper preparation. J.Z. designed the study, supervised the experiments, and revised the manuscript.

\section{Competing interests}

The authors declare no competing interests.

\section{Additional information}

Supplementary information is available for this paper at https://doi.org/10.1038/s41467020-18519-0.

Correspondence and requests for materials should be addressed to J.Z.

Peer review information Nature Communications thanks Gary Lopaschuk, Hongliang Li and the other, anonymous, reviewer(s) for their contribution to the peer review of this work.

Reprints and permission information is available at http://www.nature.com/reprints

Publisher's note Springer Nature remains neutral with regard to jurisdictional claims in published maps and institutional affiliations.

Open Access This article is licensed under a Creative Commons Attribution 4.0 International License, which permits use, sharing, adaptation, distribution and reproduction in any medium or format, as long as you give appropriate credit to the original author(s) and the source, provide a link to the Creative Commons license, and indicate if changes were made. The images or other third party material in this article are included in the article's Creative Commons license, unless indicated otherwise in a credit line to the material. If material is not included in the article's Creative Commons license and your intended use is not permitted by statutory regulation or exceeds the permitted use, you will need to obtain permission directly from the copyright holder. To view a copy of this license, visit http://creativecommons.org/ licenses/by/4.0/.

(C) The Author(s) 2020 